\title{
A systematic review of nonrandomized controlled trials on the curative effects of aquatic exercise
}

This article was published in the following Dove Press journal:

International Journal of General Medicine

24 March 20II

Number of times this article has been viewed

\section{Hiroharu Kamioka' \\ Kiichiro Tsutani² \\ Yoshiteru Mutoh ${ }^{3}$ \\ Hiroyasu Okuizum ${ }^{4}$ \\ Miho Ohta ${ }^{5}$ \\ Shuichi Handa ${ }^{4}$ \\ Shinpei Okada ${ }^{6}$ \\ Jun Kitayuguchi ${ }^{7}$ \\ Masamitsu Kamada ${ }^{7}$ \\ Nobuyoshi Shiozawa ${ }^{8}$ \\ Sang-Jun Park ${ }^{4}$ \\ Takuya Honda ${ }^{4}$ \\ Shoko Moriyama ${ }^{4}$ \\ 'Faculty of Regional Environment Science, Tokyo University of Agriculture, Tokyo, Japan; ${ }^{2}$ Department of Drug Policy and Management, Graduate School of Pharmaceutical Sciences, ${ }^{3}$ Department of Physical and Health Education, Graduate School of Education, The University of Tokyo, Tokyo, Japan; ${ }^{4}$ Mimaki Onsen (Spa) Clinic, Tomi City, Japan; ${ }^{5}$ Laboratory of Aqua, Health, and Sports Medicine, ${ }^{6}$ Physical Education and Medicine Research Foundation, Nagano, Japan; ${ }^{7}$ Physical Education and Medicine Research Center Unnan, Unnan City, Japan; ${ }^{8}$ Department of Longevity and Social Medicine, Okayama University Graduate School of Medicine, Dentistry and Pharmaceutical Sciences, Okayama, Japan}

Correspondence: Hiroharu Kamioka Faculty of Regional Environment Science, Tokyo University of Agriculture, I-I-I Sakuragaoka, Setagaya-ku,

Tokyo 156-8502, Japan

Tel/fax+8I 354772587

Email hlkamiok@nodai.ac.jp
Background: The objectives of this review were to integrate the evidence of curative effects through aquatic exercise and assess the quality of studies based on a review of nonrandomized controlled trials (nRCTs).

Methods: Study design was a systematic review of nonrandomized controlled trials. Trials were eligible if they were nonrandomized clinical trials. Studies included one treatment group in which aquatic exercise was applied. We searched the following databases from 2000 up to July 20, 2009: MEDLINE via PubMed, CINAHL, and Ichushi-Web.

Results: Twenty-one trials met all inclusion criteria. Languages included were English ( $N=9$ ), Japanese $(\mathrm{N}=11)$, and Korean $(\mathrm{N}=1)$. Target diseases were knee and/or hip osteoarthritis, poliomyelitis, chronic kidney disease, discomforts of pregnancy, cardiovascular diseases, and rotator cuff tears. Many studies on nonspecific disease (healthy participants) were included. All studies reported significant effectiveness in at least one or more outcomes. However results of evaluations with the TREND and CLEAR-NPT checklists generally showed a remarkable lack of description in the studies. Furthermore, there was the problem of heterogeneity, and we were therefore not able to perform a meta-analysis.

Conclusion: Because there was insufficient evidence on aquatic exercise due to poor methodological and reporting quality and heterogeneity of nRCTs, we were unable to offer any conclusions about the effects of this intervention. However, we were able to identify problems with current nRCTs of aquatic exercise, and propose a strategy of strengthening study quality, stressing the importance of study feasibility as a future research agenda objective.

Keywords: aquatic exercise, systematic review, nonrandomized controlled trials

\section{Introduction}

Over the years, aquatic exercise has been known as pool therapy, hydrotherapy, and sometimes in earlier literature, as balneotherapy. ${ }^{1}$ Exercise in warm water, usually termed hydrotherapy or aquatic therapy, is a popular treatment with a pain relief effect for many patients with painful neurologic or musculoskeletal conditions. ${ }^{2}$ The warmth and buoyancy of water may block nociception by acting on thermal receptors and mechanoreceptors, thus influencing spinal segmental mechanisms. ${ }^{3,4}$ In addition, the warmth may enhance blood flow, which is thought to help in dissipating algogenic chemicals, and it may facilitate muscle relaxation. The hydrostatic effect may also relieve pain by reducing peripheral edema ${ }^{5}$ and by dampening sympathetic nervous system activity. ${ }^{6}$

Recent reports have demonstrated the effectiveness of comprehensive health education, including lifestyle education and exercise in combination with spa bathing, 
for male white-collar workers, ${ }^{7}$ and middle-aged and elderly people..$^{8,9}$

It is well known in research design that evidence grading is highest for a systematic review (SR) with meta-analysis of randomized controlled trials (RCTs). In "the recent review (summary) ${ }^{10}$ of the SRs of RCTs", it was reported that there were three $\mathrm{SRs}^{1,2,11}$ that included meta-analyses of RCTs on aquatic exercise. Bartels et $\mathrm{al}^{1}$ reported that aquatic exercise had some beneficial short-term effects for patients with hip and/or knee osteoarthritis. Hall et $\mathrm{al}^{2}$ reported that aquatic exercise had a small post-treatment effect in relieving pain compared with no treatment for patients with neurologic and musculoskeletal diseases, but there were no differences in pain relieving effects between aquatic and land exercise. Pittler et $\mathrm{al}^{11}$ suggested that spa exercise may be effective for treating patients with chronic low back pain. However, we did not find any SRs of RCTs in which physical (eg, cardiovascular fitness) or psychological (eg, depression) effects were the primary outcome measurements.

An RCT is initially very difficult to execute and contains etiological issues, while the design of a non-RCT (nRCT) is easy to implement compared with an RCT. Although many studies have reported the curative effects of locomotrium diseases through aquatic exercise, there have been no systematic reviews of the evidence based on nRCTs. The objective of this study was to integrate the evidence from nRCTs on the curative effects through aquatic exercise for various diseases, and to assess the quality of those trials.

\section{Methods}

\section{Criteria for considering studies included in this study}

Studies were eligible if they were nRCTs and included one treatment group in which curative aquatic exercise was applied. Any type of aquatic exercise for cure and not for sports (eg, swimming) was permitted. The use of medication, alternative therapies, or lifestyle changes was described, and had to have been comparable in the group studies. There was no restriction on language.

\section{Search methods for identification of studies}

We searched the following databases from 2000 up to July 20, 2009: MEDLINE via PubMed, CINAHL, Web of Science, and Ichushi-Web (in Japanese). The International Committee of Medical Journal Editors (ICMJE) recommended uniform requirements for manuscripts submitted to biomedical journals in 1993. We selected articles published on and after 2000 because it appeared that the ICMJE recommendation had been adopted by the relevant researchers and had strengthened the quality of reports.

All searches were performed by two specific searchers (hospital librarians) who were qualified in medical information handling, and who were sophisticated in clinical trial research.

\section{Search strategies}

The search strategies contained the following elements and terms for all databases:

I: Search "aquatic exercise" or "water exercise"

II: Search "water gymnastic" or "water aerobics" or "pool exercise" or "pool therapy" or "aerobic aquatics" or aquatics

III: Search "exercise therapy"[MeSH] and "water" [MeSH]

IV: Search "water-based exercise"[All Fields] or "waterbased training"[All Fields] or "aquatic therapy"[All Fields] or "aquatic physical therapy"[All Fields] or "water training”[All Fields] or "water-gymnastics"[All Fields]

V: Search I or II or III or IV Limits: Publication Date from January 1, 2000 to 2009

VI: Search I or II or III or IV Limits: Publication Date from January 1, 2000 to 2009, Randomized Controlled Trial VII: Search V not VI.

Only keywords about intervention were used for the searches. First, titles and abstracts of identified published articles were reviewed to determine the relevance of the articles. Next, references in relevant studies and identified nRCTs were screened.

2000 is the year the CONSORT Statement became available on the Internet. The CONSORT Statement was created in the mid-1990s for improving the quality of RCTs. Because of the impact of the Internet, the quality of RCTs has improved since 2000 .

\section{Reference checking, hand-searching and others}

We did not check the references of included studies, perform any hand-searching, or contact any institutions, societies, or specialists known to have expertise in aquatic exercise, or authors of included studies to identify any additional published or unpublished data.

\section{Selection of trials}

To make the final selection of studies for the review, all criteria were applied independently by two authors (JK and NS) to 
the full text of articles that had passed the first eligibility screening (Figure 1). Disagreements and uncertainties were resolved by discussion between the review authors.

Studies were selected when 1) the design was an nRCT and 2) one of the interventions was a form of aquatic exercise. Curative effects were used as a primary outcome measure. Trials that were excluded are presented with reasons for exclusion (Appendix 1).

\section{Quality assessment and summary of studies}

To ensure that variation was not caused by systematic errors in the study design or execution, two review authors (MK and HK) independently assessed the quality of articles. A full quality appraisal of these papers was made using the TREND statement checklist ${ }^{12}$ and CLEAR-NPT checklist, ${ }^{13}$ developed to assess the methodological quality of nRCTs

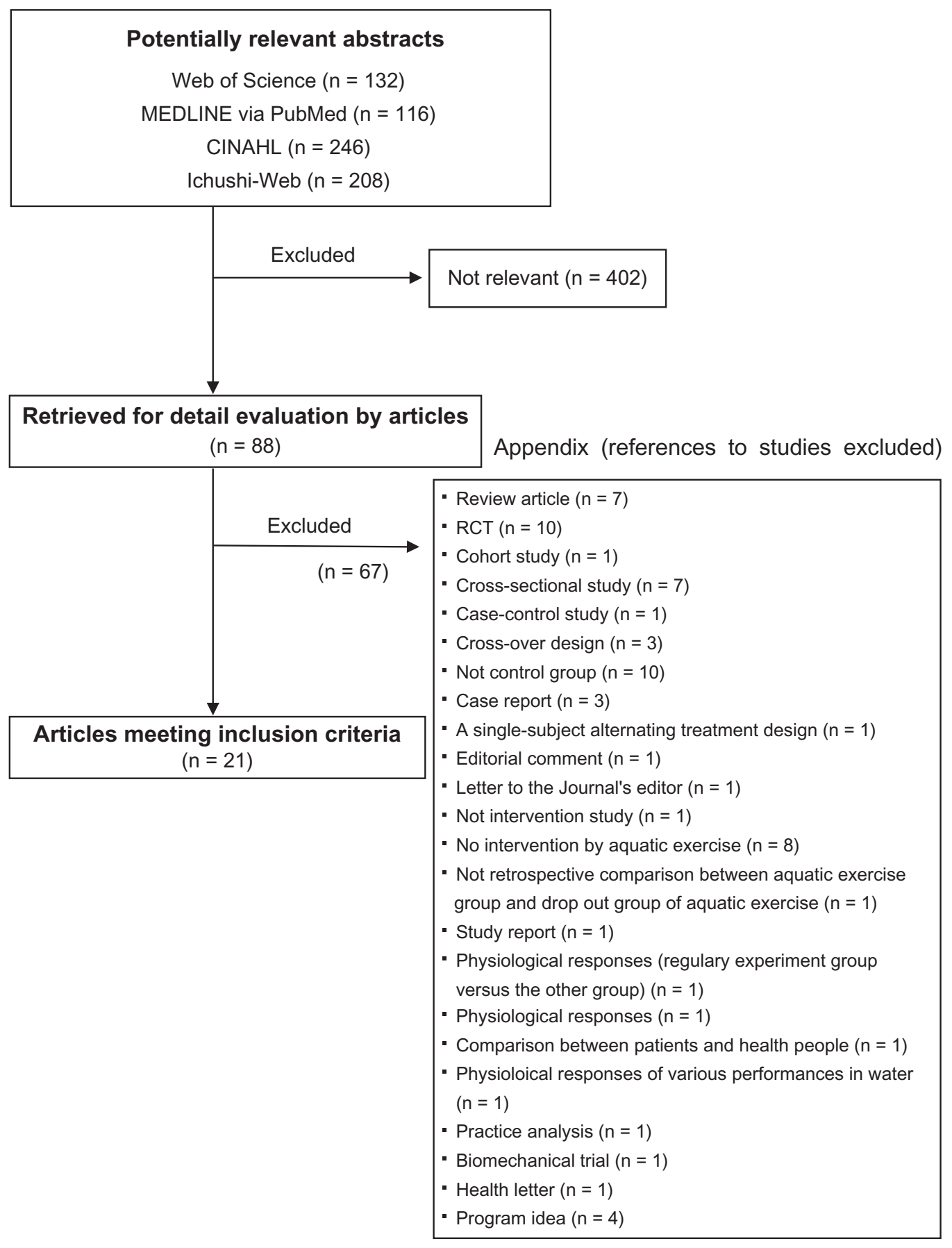

Figure I Flowchart of trial process.

Abbreviation: RCT, randomized controlled trial. 
and nonpharmacological trials, respectively. Disagreements and uncertainties were resolved by discussion between the review authors.

For meta-analysis preparation, the target objects and main outcomes in each study were examined. We found that there were various kinds of target diseases in the studies reviewed: healthy young students, middle-aged or elderly people, or people with a certain disease. In addition, the studies were heterogeneous, and the main outcomes varied. Moreover, the quality of most studies was low according to the checklist results, and such low-quality studies were excluded from the analysis based on the Cochrane Review. ${ }^{1}$ We could not perform a meta-analysis since no variable was eligible.

One review author (HK) selected the summary from each of the structured abstracts.

\section{Benefit, harm, and withdrawals}

The GRADE Working Group ${ }^{14}$ reported that the balance between benefit and harm, quality of evidence, applicability, and the certainty of the baseline risk were all considered in judgments about the strength of recommendations. Adverse events, withdrawals, and the cost for intervention were especially important information for researchers and users of clinical practice guidelines, and we presented this information with the description of each article.

\section{Results}

\section{Study characteristics}

The literature searches included 402 potentially relevant articles (Figure 1). Abstracts from those articles were assessed and 88 papers were retrieved for further evaluation (checks for relevant literature). Sixty-seven publications were excluded because they did not meet the eligibility criteria (see Appendix 1). Twenty-one trials ${ }^{15-35}$ met all inclusion criteria (Table 1). The languages of the eligible publications were English $(\mathrm{N}=9)$, Japanese $(\mathrm{N}=11)$, and Korean $(\mathrm{N}=1)$. Target diseases were knee and/or hip osteoarthritis, ${ }^{19,24,28}$ poliomyelitis, ${ }^{15}$ chronic kidney disease, ${ }^{21}$ discomforts of pregnancy, ${ }^{30}$ cardiovascular diseases, ${ }^{33}$ and rotator cuff tears. $^{35}$ Many studies ${ }^{16-18,20-23,25-27,29,31,32,34}$ on nonspecific disease (healthy participants) were included (Table 2). All studies reported significant effectiveness in one or more outcomes. In particular, many studies reported that aquatic exercise had a significant effect on pain relief and outcome measurements for locomotor diseases..$^{15,19,24,28,35}$ These intervention periods ranged from 2 weeks to 12 months. These reflected the difficulty of maintaining long-term participation in each intervention trial. Whatever the case, the long-term effects are not clear.

\section{Withdrawals and adverse events}

Withdrawals (dropouts) were reported in five studies, ${ }^{24,28,29,32,34}$ and adverse events were reported in four studies (Table 2). There were three studies ${ }^{15,19,35}$ that reported 'nothing' on adverse events, and one study ${ }^{28}$ reported a slipping accident on the poolside (details of the injury were unclear). Other studies did not provide information on withdrawals or adverse events.

\section{Intervention costs}

A description of intervention costs was included in only one trial, ${ }^{30}$ but the summary of that trial did not describe the costs (Table 2).

\section{Quality assessment}

We evaluated 21 items from the TREND checklist in more detail (Table 3 ). This assessment evaluated the quality of how the main findings of the study were summarized in the written report. A lack of description was noteworthy for the studies in general. The items for which the description rate was less than $30 \%$ were as follows: "information on how units were allocated to interventions (23.8\%)"; "how sample size was determined and, when applicable, explanation of any interim analyses and stopping rules (23.8\%)"; "method used to assign units to study conditions, including details of any restriction (19.0\%)"; "inclusion of aspects employed to help minimize potential bias induced due to non-randomization (4.8\%)"; "whether or not participants, those administering the interventions, and those assessing the outcomes were blinded to study condition assignment; if so, statement regarding how the blinding was accomplished and how it was assessed (14.3\%)"; "if the unit of analysis differs from the unit of assignment, the analytical method used to account for this (9.5\%)"; "statistical methods used for additional analyses, such as subgroup analyses and adjusted analysis (9.5\%)"; "methods for imputing missing data, if used (14.3\%)"; "flow of participants through each stage of the study: enrollment, assignment, allocation and intervention exposure, follow-up, analysis (19.0\%)"; "dates defining the periods of recruitment and follow-up (14.3\%)"; "baseline comparisons of those lost to follow-up and those retained, overall and by study condition (9.5\%)"; "comparison between study population at baseline and target population of interest (4.8\%)"; "indication of whether the analysis strategy was 'intention to treat' or, if not, description of how noncompliers were treated in the 


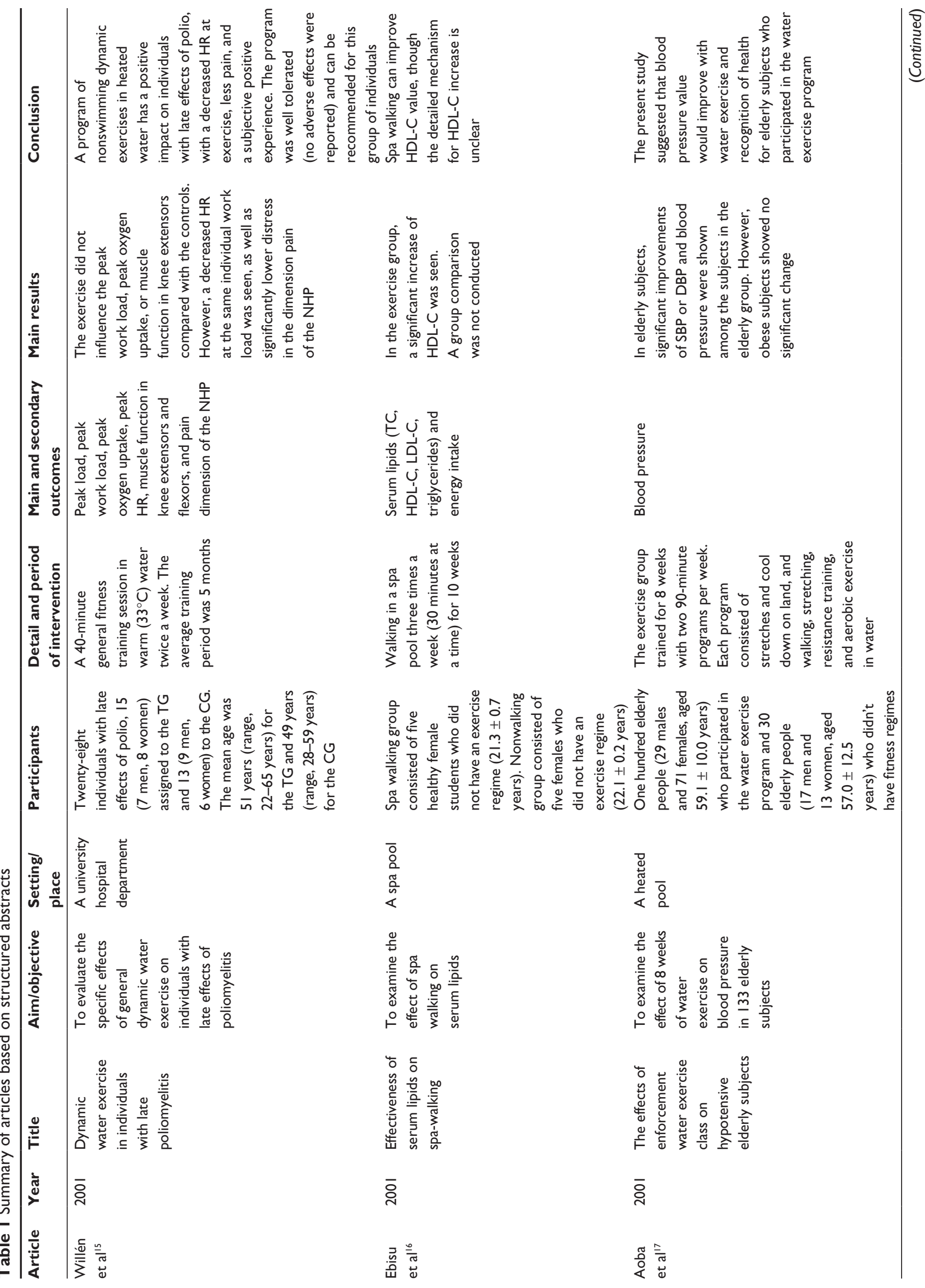




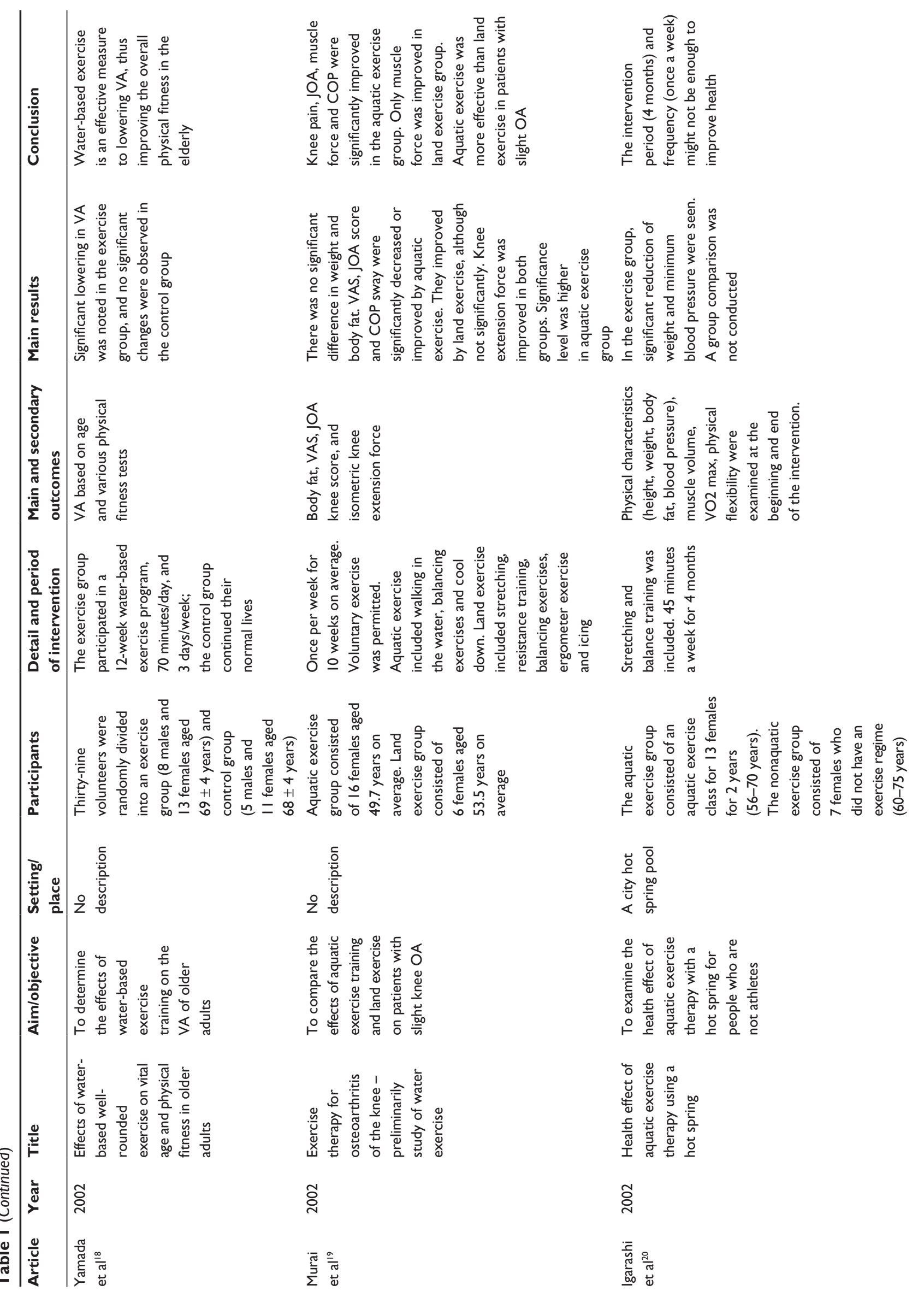



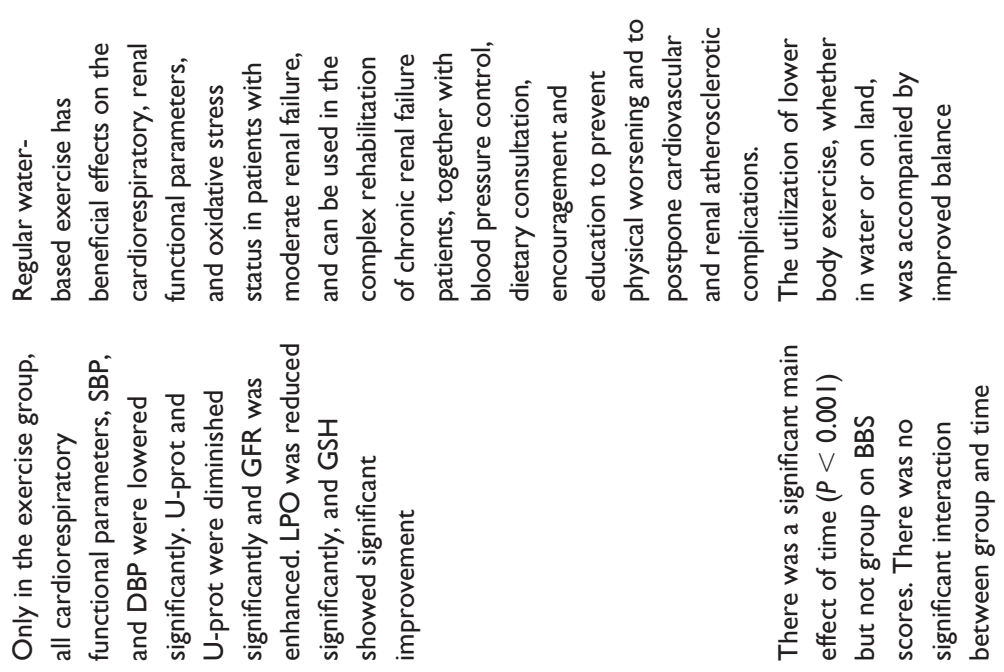

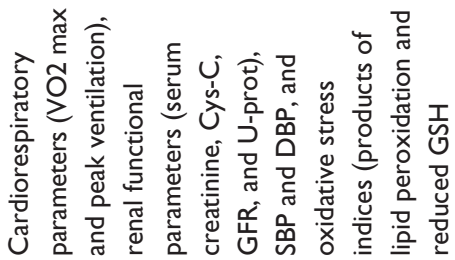

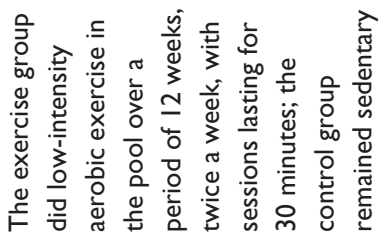

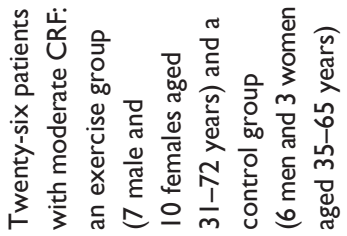

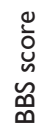

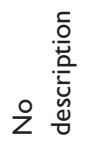

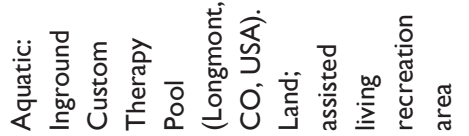

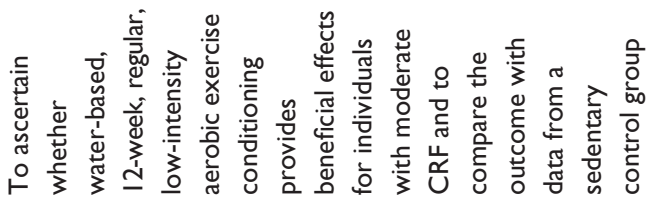
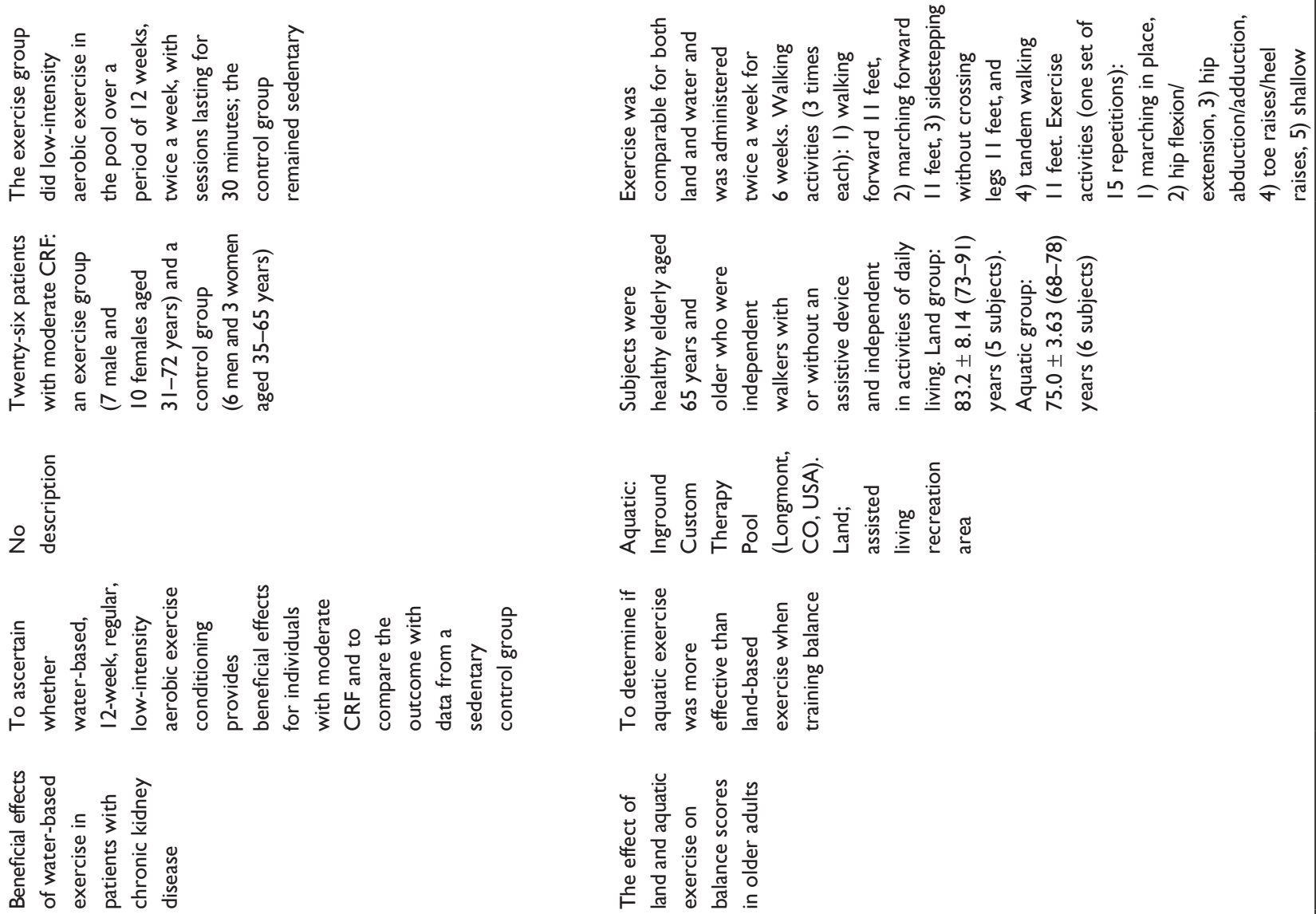

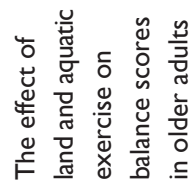

ळั.

ڤ̊ํํ

这离

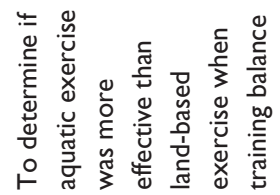




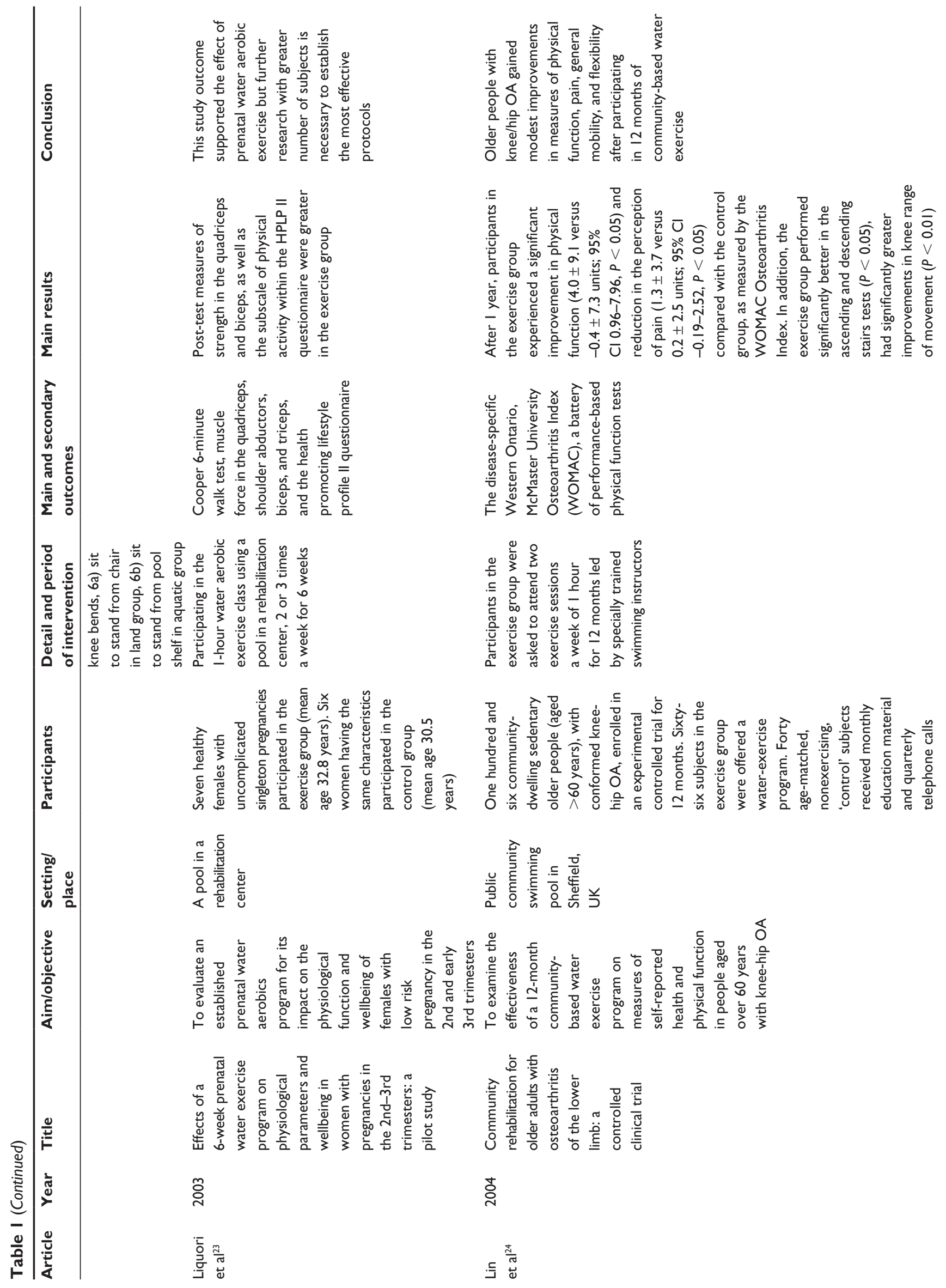




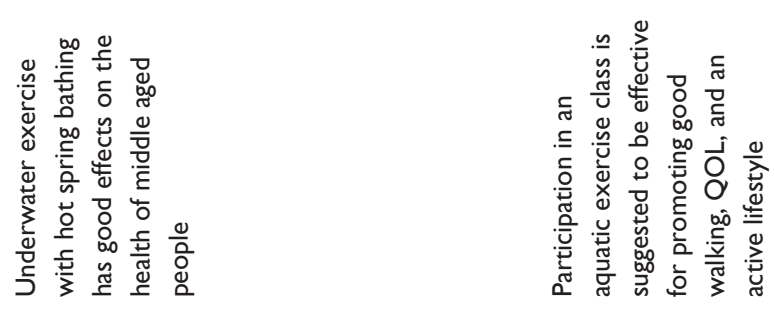

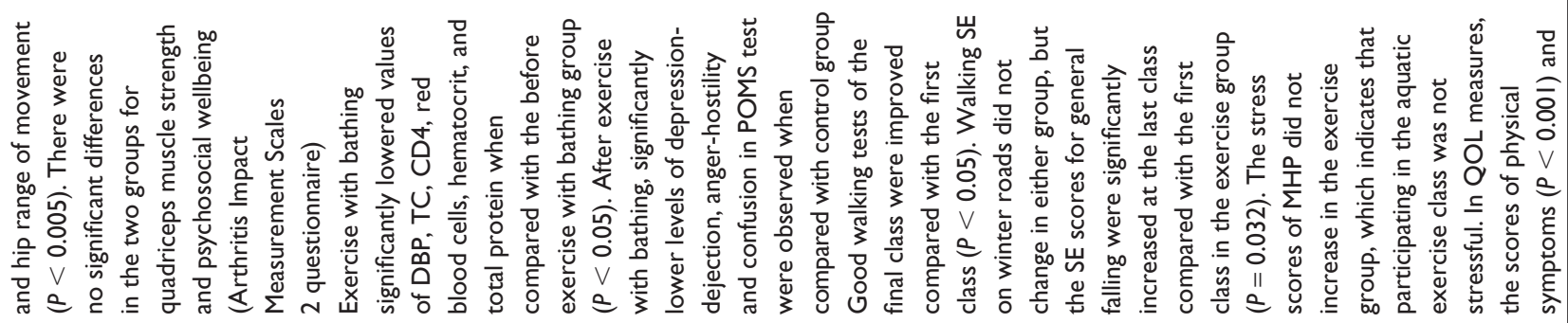
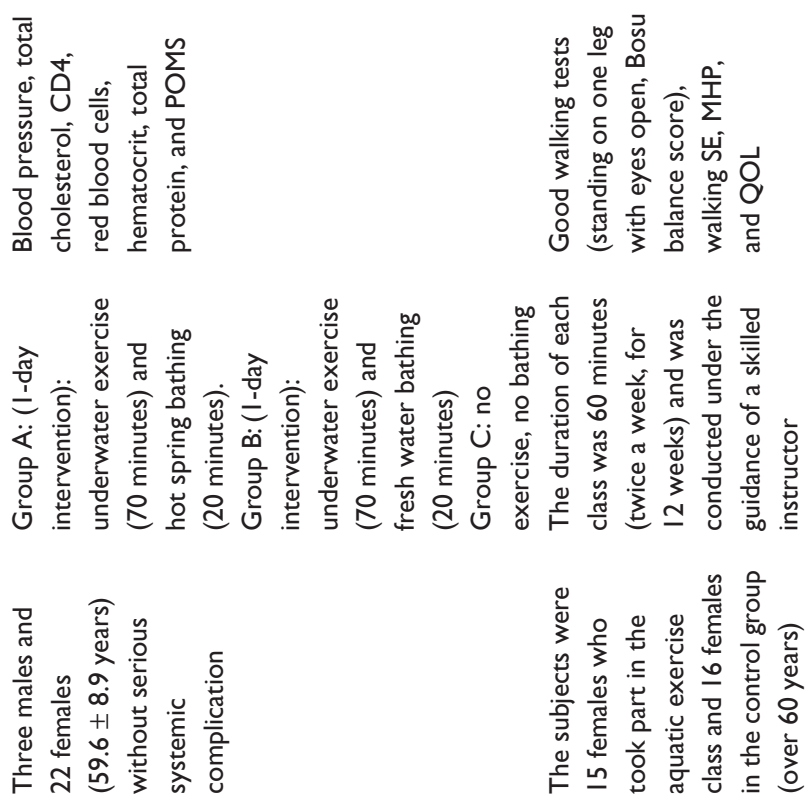

z을ำ
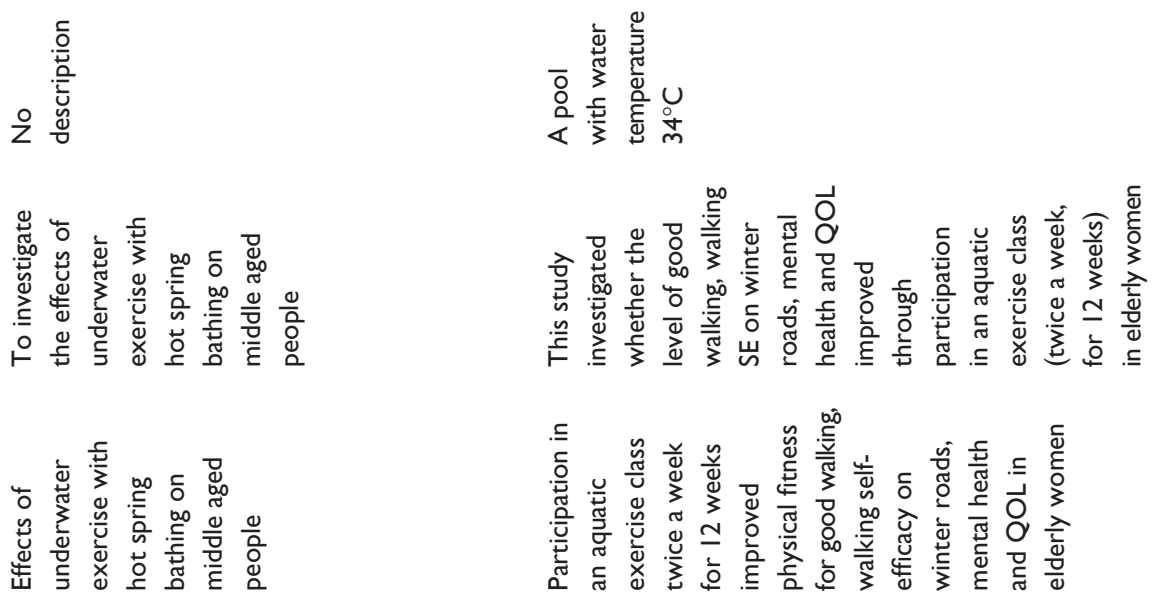

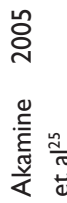

옹

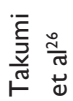




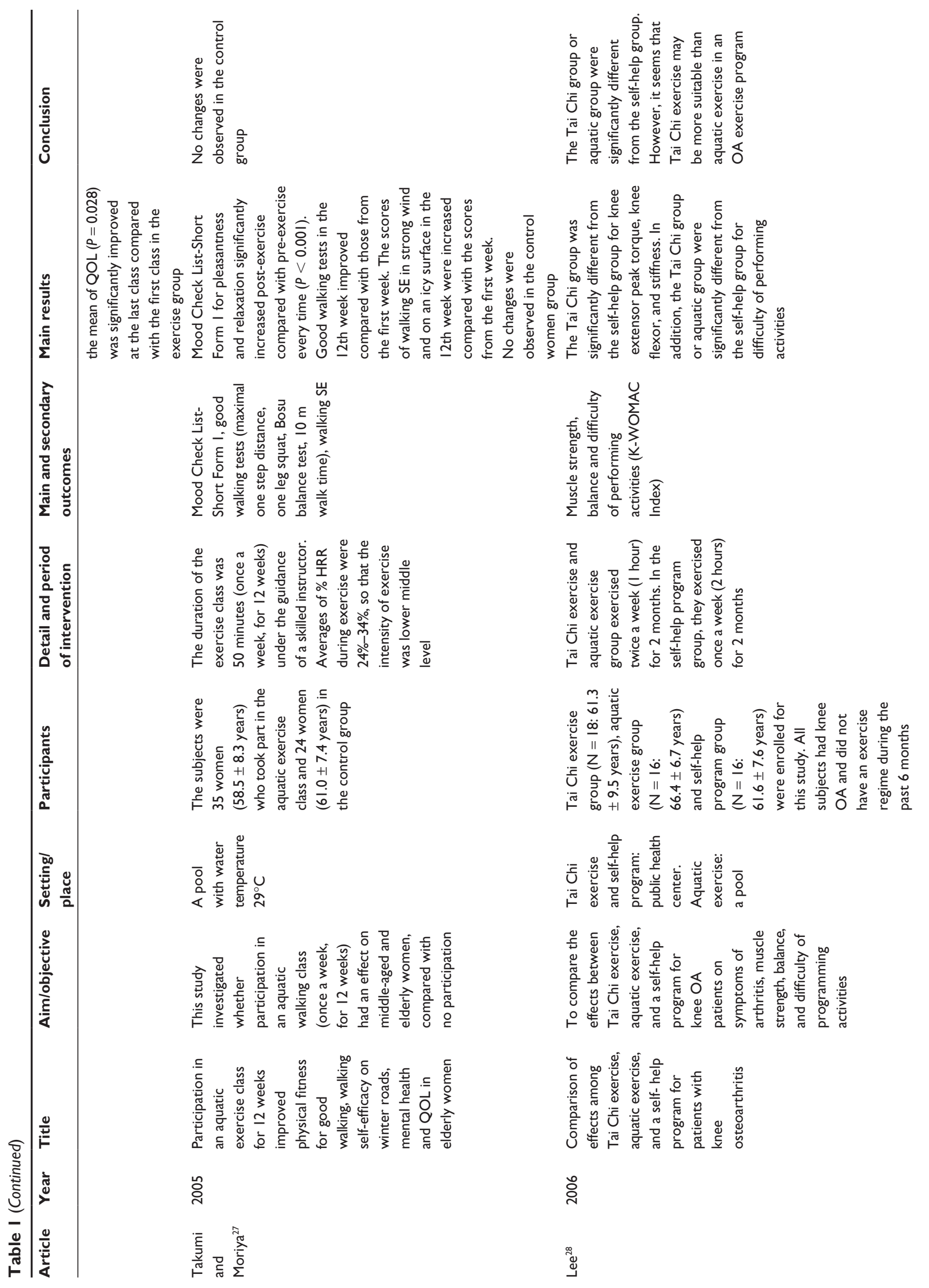



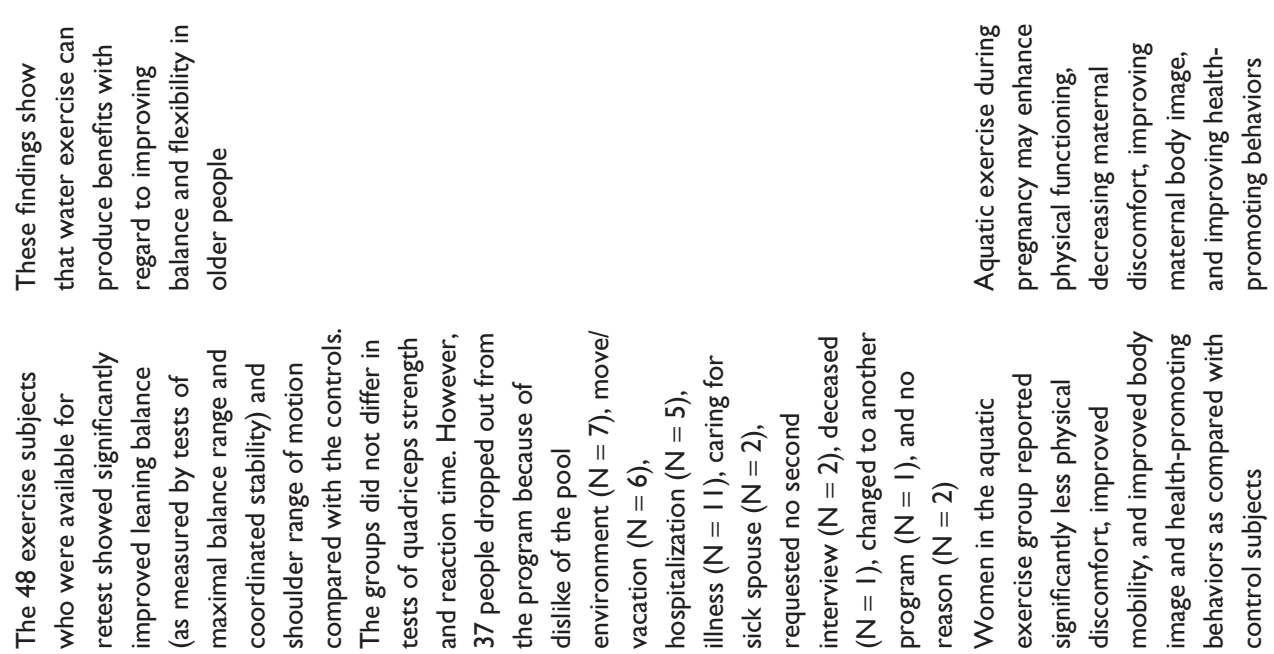

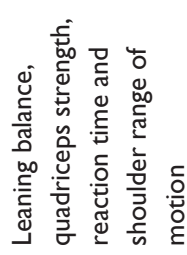

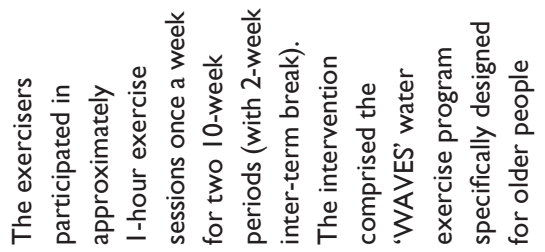

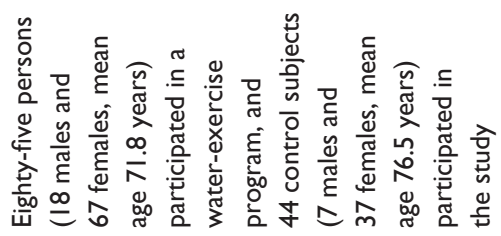

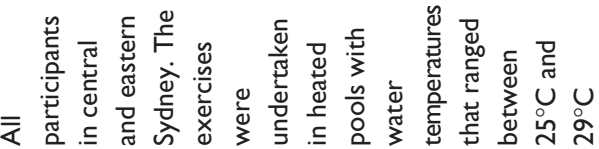

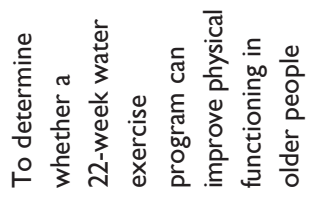

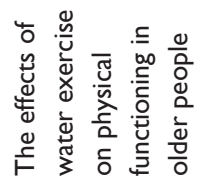

ठั่

ำ

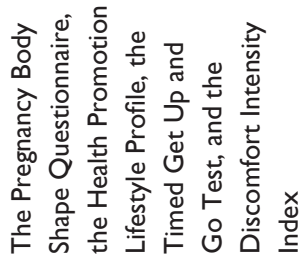

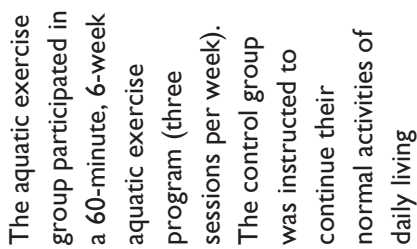

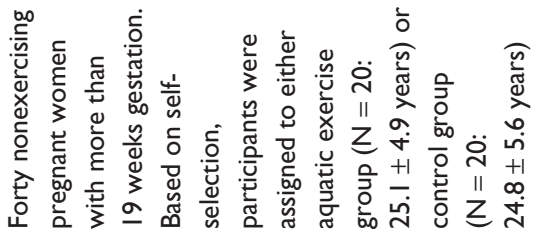

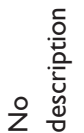

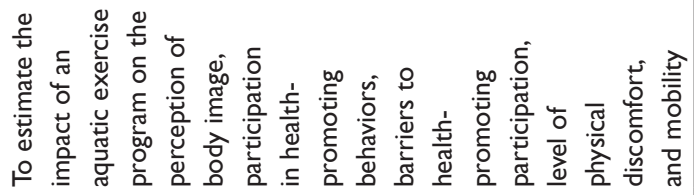

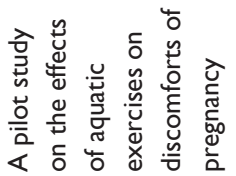

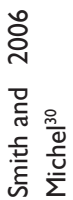




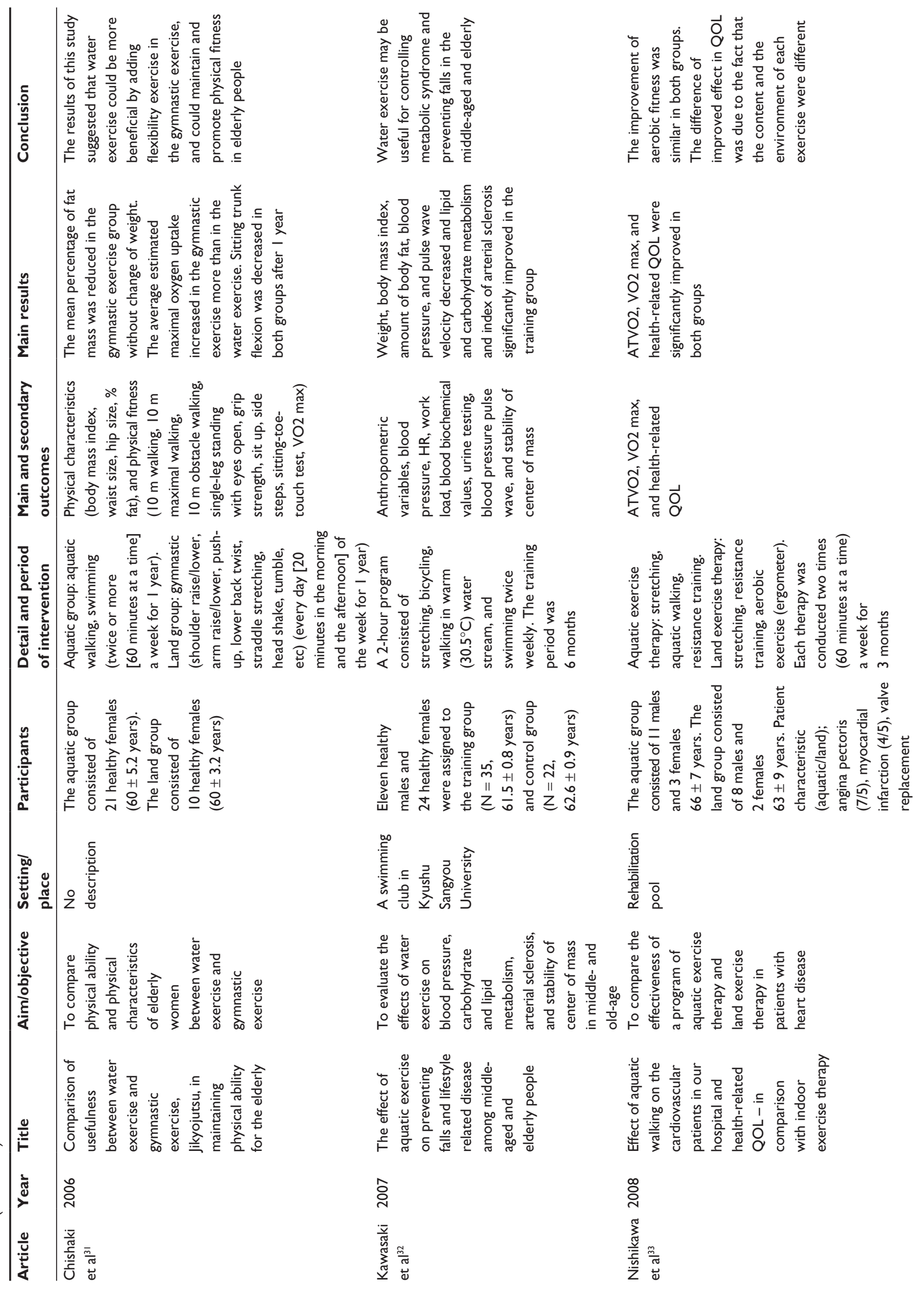



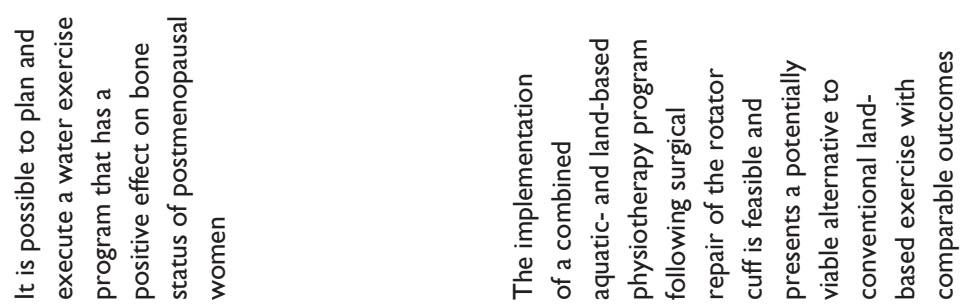

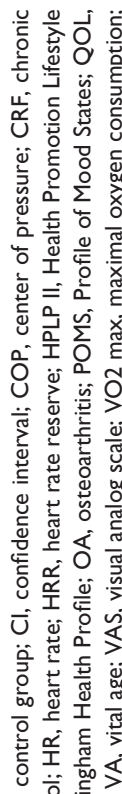

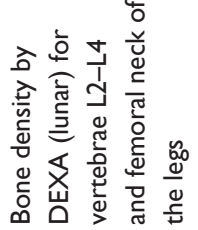

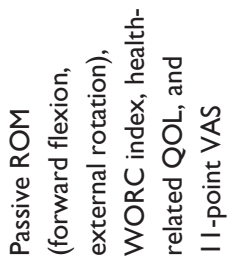

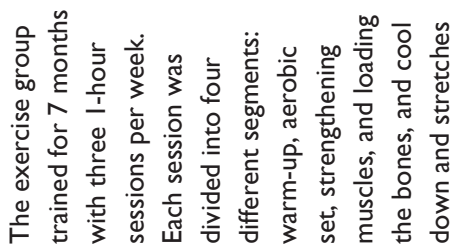
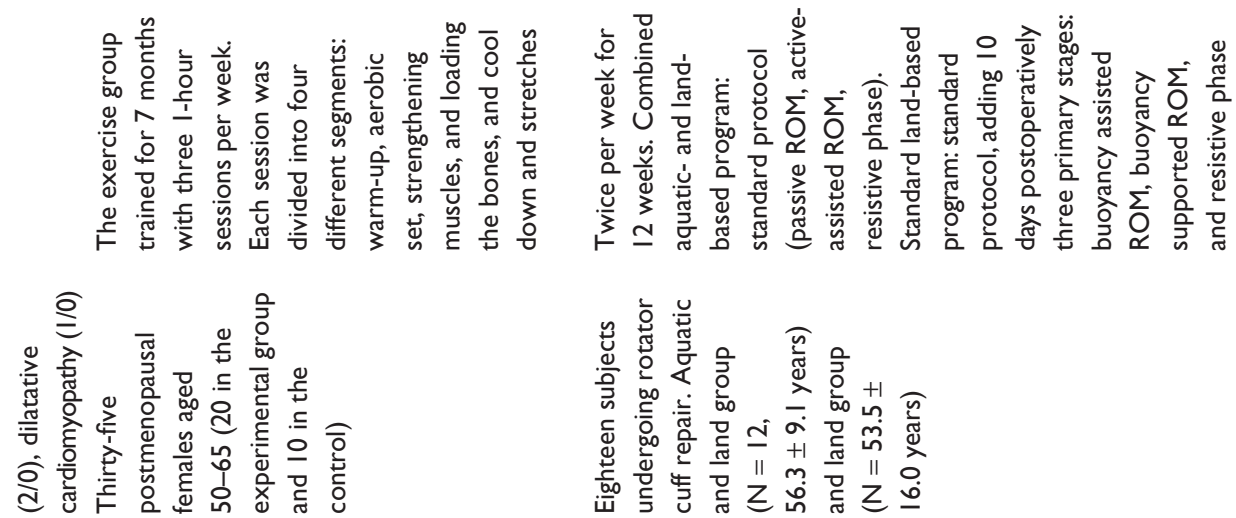

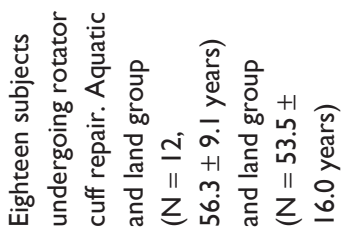

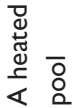

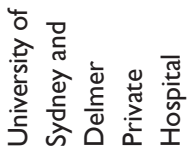

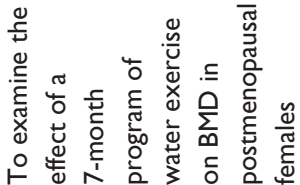

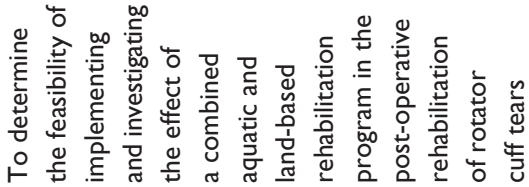

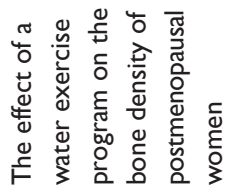

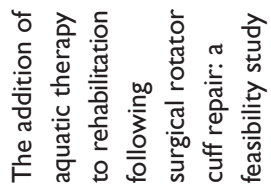

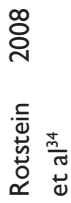

ঃั

穿第

ปै

言运产。

政它

달 응

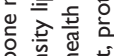

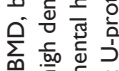

苞它全

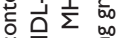

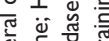

을

o

5 茄

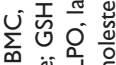

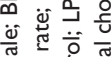

要

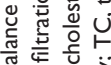

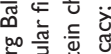

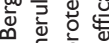

空

话

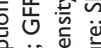

言立能

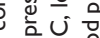

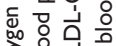

당 응

进 믕 능 尊

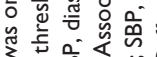

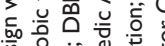

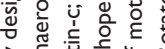

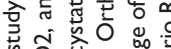

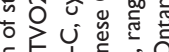

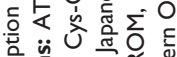

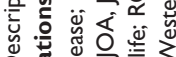

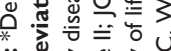

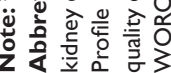


Table 2 Brief summary of 21 articles

\begin{tabular}{|c|c|c|c|c|c|c|}
\hline Article & $\begin{array}{l}\text { Year of } \\
\text { publication }\end{array}$ & Object disease & Effects noted & Withdrawals & Adverse event & Cost of intervention \\
\hline Willén et al ${ }^{15}$ & 2001 & Poliomyelitis & Significant effect & No withdrawal & Nothing & No description \\
\hline Ebisu et $\mathrm{al}^{16}$ & 2001 & No specific disease & Significant effect ${ }^{\mathrm{a}}$ & No description & No description & No description \\
\hline Aoba et $\mathrm{al}^{17}$ & 2001 & No specific disease & Significant effect & No description & No description & No description \\
\hline Yamada et al ${ }^{18}$ & 2002 & No specific disease & Significant effect & No description & No description & No description \\
\hline Murai et al ${ }^{19}$ & 2002 & Knee OA & Significant effect & No description & Nothing & No description \\
\hline Igarashi et $\mathrm{al}^{20}$ & 2002 & No specific disease & Significant effect ${ }^{a}$ & No description & No description & No description \\
\hline Pechter et $\mathrm{al}^{2 \mathrm{I}}$ & 2003 & $\begin{array}{l}\text { Moderate chronic } \\
\text { kidney disease }\end{array}$ & Significant effect & No description & No description & Not description \\
\hline Douris et $\mathrm{a}^{22}$ & 2003 & No specific disease & Significant effect & No description & No description & Not description \\
\hline Liquori et $\mathrm{a}^{23}$ & 2003 & No specific disease & Significant effect & No description & No description & No description \\
\hline Lin et $\mathrm{al}^{24}$ & 2004 & Knee-hip OA & Significant effect & $\begin{array}{l}\mathrm{N}=9 ; \text { Reason } \\
\text { was not described. }\end{array}$ & No description & No description \\
\hline Akamine et $\mathrm{a}^{25}$ & 2005 & No specific disease & Significant effect & No description & No description & No description \\
\hline Takumi et $\mathrm{a}^{26}$ & 2005 & No specific disease & Significant effect & No description & No description & No description \\
\hline $\begin{array}{l}\text { Takumi and } \\
\text { Moriya }^{27}\end{array}$ & 2005 & No specific disease & Significant effect & No description & No description & No description \\
\hline $\operatorname{Lee}^{28}$ & 2006 & Knee OA & Significant effect & $\begin{array}{l}\mathrm{N}=3 \text {; Slipping on } \\
\text { poolside, having a } \\
\text { cold, low height. }\end{array}$ & $\begin{array}{l}\mathrm{N}=\text { I Slipping } \\
\text { on poolside. }\end{array}$ & No description \\
\hline Lord et $\mathrm{a}^{29}$ & 2006 & No specific disease & Significant effect & $\begin{array}{l}N=37 ; \text { Reasons } \\
\text { were shown in } \\
\text { Table I in detail. }\end{array}$ & No description & No description \\
\hline $\begin{array}{l}\text { Smith and } \\
\text { Michel }^{30}\end{array}$ & 2006 & $\begin{array}{l}\text { Discomforts } \\
\text { of pregnancy }\end{array}$ & Significant effect & No description & No description & $\begin{array}{l}\text { After pretests were } \\
\text { completed, each } \\
\text { participant received a } \\
\$ 10 \text { grocery store gift } \\
\text { certificate, and each } \\
\text { one in the aquatic } \\
\text { exercise group were } \\
\text { given the bus tickets } \\
\text { or parking passes to } \\
\text { assist participants' } \\
\text { attendance. Other } \\
\text { cost was not described }\end{array}$ \\
\hline Chishaki et $\mathrm{al}^{31}$ & 2006 & No specific disease & Significant effect & No description & No description & No description \\
\hline Kawasaki et a ${ }^{32}$ & 2007 & No specific disease & Significant effect & $\begin{array}{l}\mathrm{N}=8 ; \text { Low } \\
\text { compliance. }\end{array}$ & No description & No description \\
\hline Nishikawa et a ${ }^{33}$ & 2008 & $\begin{array}{l}\text { Cardiovascular } \\
\text { disease }\end{array}$ & Significant effect & No withdrawal & No description & No description \\
\hline Rotstein et $\mathrm{al}^{34}$ & 2008 & No specific disease & Significant effect & $\begin{array}{l}\mathrm{N}=5 ; \text { Reason was } \\
\text { not described. }\end{array}$ & No description & No description \\
\hline Brady et $\mathrm{al}^{35}$ & 2008 & Rotator cuff tears & Significant effect & No description & Nothing & No description \\
\hline
\end{tabular}

Note: aGroup comparison was not conducted.

Abbreviation: OA, osteoarthritis.

analyses (9.5\%)"; "for each primary and secondary outcome, a summary of results for each study condition, and the estimated effect (9.5\%)"; "inclusion of results from testing prespecified causal pathways through which the intervention was intended to operate, if any (14.3\%)"; "summary of other analyses performed, including subgroup or restricted analyses, indicating which are prespecified or exploratory (4.8\%)"; and "summary of all important adverse events or unintended effects in each study condition (19.0\%)".
Table 4 presents an assessment of the evaluation of study quality by use of the CLEAR-NPT checklist. This tool mainly evaluated the quality of the study conduct. The description rate for the details of the intervention was high (81\%), but the rate was low in other primary items. The primary items for which the executive rate was less than $30 \%$ were as follows: "Were participants adequately blinded? (4.8\%)"; "Were care providers or persons adequately blinded? (4.8\%)"; "Were outcome assessors adequately blinded to assess the primary 
Table 3 Evaluation of the quality of nonrandomized controlled trials by using the TREND checklist

\begin{tabular}{|c|c|c|c|c|}
\hline Paper section/topic & Item no. & Descriptor & $\mathbf{N}$ of yes & $\%$ \\
\hline \multirow[t]{3}{*}{ Title and abstract } & 1 & - Information on how units were allocated to interventions & $5 / 21$ & 23.8 \\
\hline & & - Structured abstract recommended & $8 / 21$ & 38.1 \\
\hline & & - Information on target population or study sample & $12 / 2 \mid$ & 57.1 \\
\hline \multicolumn{5}{|l|}{ Introduction } \\
\hline \multirow[t]{2}{*}{ Background } & 2 & - Scientific background and explanation of rationale & $|8 / 2|$ & 85.7 \\
\hline & & - Theories used in designing behavioral interventions & $8 / 21$ & 38.1 \\
\hline \multicolumn{5}{|l|}{ Methods } \\
\hline \multirow[t]{3}{*}{ Participants } & 3 & $\begin{array}{l}\text { - Eligibility criteria for participants, including criteria at different levels in } \\
\text { recruitment/sampling plan (eg, cities, clinics, subjects) }\end{array}$ & $|4 / 2|$ & 66.7 \\
\hline & & $\begin{array}{l}\text { - Method of recruitment (eg, referral, self-selection), including the sampling } \\
\text { method if a systematic sampling plan was implemented }\end{array}$ & $10 / 21$ & 47.6 \\
\hline & & - Recruitment setting (settings and locations where the data were collected) & $7 / 21$ & 33.3 \\
\hline \multirow[t]{9}{*}{ Interventions } & 4 & $\begin{array}{l}\text { - Details of the interventions intended for each study condition and how } \\
\text { and when they were actually administered, specifically including: }\end{array}$ & ||$/ 2 \mid$ & 52.4 \\
\hline & & - Content: what was given? & $19 / 21$ & 90.5 \\
\hline & & - Delivery method: how was the content given? & $19 / 21$ & 90.5 \\
\hline & & - Unit of delivery: how were subjects grouped during delivery? & $|3 / 2|$ & 61.9 \\
\hline & & - Deliverer: who delivered the intervention? & $10 / 21$ & 47.6 \\
\hline & & - Setting: where was the intervention delivered? & $16 / 21$ & 76.2 \\
\hline & & $\begin{array}{l}\text { - Exposure quantity and duration: how many sessions or episodes or events } \\
\text { were intended to be delivered? How long were they intended to last? }\end{array}$ & $20 / 21$ & 95.2 \\
\hline & & - Time span: how long was it intended to deliver the intervention to each unit? & $21 / 21$ & 100.0 \\
\hline & & - Activities to increase compliance or adherence (eg, incentives) & $7 / 21$ & 33.3 \\
\hline Objectives & 5 & - Specific objectives and hypotheses & $|5 / 2|$ & 71.4 \\
\hline \multirow[t]{3}{*}{ Outcomes } & 6 & - Clearly defined primary and secondary outcome measures & $17 / 21$ & 81.0 \\
\hline & & $\begin{array}{l}\text { - Methods used to collect data and any methods used to enhance the quality } \\
\text { of measurements }\end{array}$ & $17 / 21$ & 81.0 \\
\hline & & - Information validated instruments such as psychometric and biometric properties & $8 / 21$ & 38.1 \\
\hline Sample size & 7 & $\begin{array}{l}\text { - How sample size was determined and, when applicable, explanation of any } \\
\text { interim analyses and stopping rules }\end{array}$ & $5 / 21$ & 23.8 \\
\hline \multirow[t]{3}{*}{ Assignment method } & 8 & $\begin{array}{l}\text { - Unit of assignment (the unit being assigned to study condition, eg, individual, } \\
\text { group, community) }\end{array}$ & $16 / 21$ & 76.2 \\
\hline & & $\begin{array}{l}\text { - Method used to assign units to study conditions, including details of any } \\
\text { restriction (eg, blocking, stratification, minimization) }\end{array}$ & $4 / 21$ & 19.0 \\
\hline & & $\begin{array}{l}\text { - Inclusion of aspects employed to help minimize potential bias induced due } \\
\text { to nonrandomization (eg, matching) }\end{array}$ & $|/ 2|$ & 4.8 \\
\hline Blinding (masking) & 9 & $\begin{array}{l}\text { Whether or not participants, those administering the interventions, and those } \\
\text { assessing the outcomes were blinded to study condition assignment; if so, } \\
\text { statement regarding how the blinding was accomplished and how it was assessed }\end{array}$ & $3 / 21$ & 14.3 \\
\hline \multirow[t]{2}{*}{ Unit of analysis } & 10 & $\begin{array}{l}\text { - Description of the smallest unit that is being analyzed to assess intervention } \\
\text { effects (eg, individual, group, or community) }\end{array}$ & $|5 / 2|$ & 71.4 \\
\hline & & $\begin{array}{l}\text { - If the unit of analysis differs from the unit of assignment, the analytical method } \\
\text { used to account for this (eg, adjusting the standard error estimates by the } \\
\text { design effect or using multilevel analysis) }\end{array}$ & $2 / 21$ & 9.5 \\
\hline \multirow[t]{4}{*}{ Statistical methods } & II & $\begin{array}{l}\text { - Statistical methods used to compare study groups for primary outcome(s), } \\
\text { including complex methods for correlated data }\end{array}$ & $20 / 21$ & 95.2 \\
\hline & & $\begin{array}{l}\text { - Statistical methods used for additional analyses, such as subgroup analyses } \\
\text { and adjusted analysis }\end{array}$ & $2 / 21$ & 9.5 \\
\hline & & - Methods for imputing missing data, if used & $3 / 21$ & 14.3 \\
\hline & & - Statistical software or programs used & $10 / 21$ & 47.6 \\
\hline \multicolumn{5}{|l|}{ Results } \\
\hline \multirow[t]{2}{*}{ Participant flow } & 12 & $\begin{array}{l}\text { - Flow of participants through each stage of the study: enrollment, assignment, allocation } \\
\text { and intervention exposure, follow-up, analysis (a diagram is strongly recommended) }\end{array}$ & $4 / 21$ & 19.0 \\
\hline & & $\begin{array}{l}\text { - Enrollment: the numbers of participants screened for eligibility, found to be } \\
\text { eligible or not eligible, declined to be enrolled, and enrolled in the study }\end{array}$ & $8 / 21$ & 38.1 \\
\hline
\end{tabular}


Table 3 (Continued)

\begin{tabular}{|c|c|c|c|c|}
\hline Paper section/topic & Item no. & Descriptor & $\mathbf{N}$ of yes & $\%$ \\
\hline & & - Assignment: the numbers of participants assigned to a study condition & $20 / 21$ & 95.2 \\
\hline & & $\begin{array}{l}\text { - Allocation and intervention exposure: the number of participants assigned to each } \\
\text { study condition and the number of participants who received each intervention }\end{array}$ & $21 / 21$ & 100.0 \\
\hline & & $\begin{array}{l}\text { - Follow-up: the number of participants who completed the follow-up or } \\
\text { did not complete the follow-up (ie, lost to follow up), by study condition }\end{array}$ & $10 / 21$ & 47.6 \\
\hline & & $\begin{array}{l}\text { - Analysis: the number of participants included in or excluded from the analysis, } \\
\text { by study condition }\end{array}$ & $|3 / 2|$ & 61.9 \\
\hline & & - Description of protocol deviations from study as planned, along with reasons & $9 / 21$ & 42.9 \\
\hline Recruitment & 13 & - Dates defining the periods of recruitment and follow-up & $3 / 21$ & 14.3 \\
\hline \multirow[t]{4}{*}{ Baseline data } & 14 & $\begin{array}{l}\text { - Baseline demographic and clinical characteristics of participants in each } \\
\text { study condition }\end{array}$ & $|5 / 2|$ & 71.4 \\
\hline & & $\begin{array}{l}\text { - Baseline characteristics for each study condition relevant to specific } \\
\text { disease prevention research }\end{array}$ & $7 / 21$ & 33.3 \\
\hline & & $\begin{array}{l}\text { - Baseline comparisons of those lost to follow-up and those retained, overall } \\
\text { and by study condition }\end{array}$ & $2 / 21$ & 9.5 \\
\hline & & - Comparison between study population at baseline and target population of interest & $|/ 2|$ & 4.8 \\
\hline Baseline equivalence & 15 & $\begin{array}{l}\text { - Data on study group equivalence at baseline and statistical methods used to } \\
\text { control for baseline differences }\end{array}$ & $10 / 21$ & 47.6 \\
\hline \multirow[t]{2}{*}{ Numbers analyzed } & 16 & $\begin{array}{l}\text { - Number of participants (denominator) included in each analysis for each study } \\
\text { condition, particularly when the denominators change for different outcomes; } \\
\text { statement of the results in absolute numbers when feasible }\end{array}$ & $|8 / 2|$ & 85.7 \\
\hline & & $\begin{array}{l}\text { - Indication of whether the analysis strategy was "intention to treat" or, } \\
\text { if not, description of how noncompliers were treated in the analyses }\end{array}$ & $2 / 21$ & 9.5 \\
\hline \multirow[t]{3}{*}{$\begin{array}{l}\text { Outcomes and } \\
\text { estimation }\end{array}$} & 17 & $\begin{array}{l}\text { - For each primary and secondary outcome, a summary of results for each } \\
\text { study condition, and the estimated effect size and a confidence interval to } \\
\text { indicate the precision }\end{array}$ & $2 / 21$ & 9.5 \\
\hline & & - Inclusion of null and negative findings & $9 / 21$ & 42.9 \\
\hline & & $\begin{array}{l}\text { - Inclusion of results from testing prespecified causal pathways through which } \\
\text { the intervention was intended to operate, if any }\end{array}$ & $3 / 21$ & 14.3 \\
\hline Ancillary analyses & 18 & $\begin{array}{l}\text { - Summary of other analyses performed, including subgroup or restricted } \\
\text { analyses, indicating which are prespecified or exploratory }\end{array}$ & $|/ 2|$ & 4.8 \\
\hline Adverse events & 19 & $\begin{array}{l}\text { - Summary of all important adverse events or unintended effects in each } \\
\text { study condition (including summary measures, effect size estimates, } \\
\text { and confidence intervals) }\end{array}$ & $4 / 21$ & 19.0 \\
\hline \multicolumn{5}{|l|}{ Discussion } \\
\hline \multirow[t]{4}{*}{ Interpretation } & 20 & $\begin{array}{l}\text { - Interpretation of the results, taking into account study hypotheses, sources } \\
\text { of potential bias, imprecision of measures, multiplicative analyses, and other } \\
\text { limitations or weaknesses of the study }\end{array}$ & $10 / 21$ & 47.6 \\
\hline & & $\begin{array}{l}\text { - Discussion of results taking into account the mechanism by which the intervention } \\
\text { was intended to work (causal pathways) or alternative mechanisms or explanations }\end{array}$ & $|5 / 2|$ & 71.4 \\
\hline & & $\begin{array}{l}\text { - Discussion of the success of and barriers to implementing the intervention, } \\
\text { fidelity if implementation }\end{array}$ & $9 / 21$ & 42.9 \\
\hline & & - Discussion of research, programmatic, or policy implications & $9 / 21$ & 42.9 \\
\hline Generalizability & 21 & $\begin{array}{l}\text { - Generalizability (external validity) of the trial findings, taking into account the study } \\
\text { population, the characteristics of the intervention, length of follow-up, incentives, } \\
\text { compliance rates, specific sites/settings involved in the study, and other contextual issues }\end{array}$ & $7 / 21$ & 33.3 \\
\hline Overall evidence & 22 & - General interpretation of the results in the context of evidence and current theory & $|2 / 2|$ & 57.1 \\
\hline
\end{tabular}

outcomes? (9.5\%)"; and "Were the main outcomes analyzed according to the intention-to-treat principle? (14.3\%)".

\section{Discussion}

\section{Overall evidence and quality assessment}

There were only $21 \mathrm{nRCT}$ s about aquatic exercise published from 2000 to July 20, 2009, possibly due to the increase in studies with an RCT design. In previous $\mathrm{SRs}^{1-3}$ of RCTs, the authors actually collected many articles that were published in 2000. Pooling of the data from nRCTs was not performed because of the heterogeneity of the studies, multiple outcome measurements, and overall poor presentation. We used the TREND and CLEAR-NPT checklists as quality assessments. There were serious problems with the 
Table 4 Evaluation of the quality of nonrandomized controlled trials by using the CLEAR-NPT checklist

\begin{tabular}{|c|c|c|c|}
\hline \multirow[t]{2}{*}{ Item } & \multicolumn{3}{|c|}{ Practice; N (\%) } \\
\hline & Yes & No & Unclear \\
\hline I. Was the generation of allocation sequences adequate? ${ }^{\mathrm{a}}$ & - & - & - \\
\hline 2. Was the treatment allocation concealed? ${ }^{\mathrm{a}}$ & - & - & - \\
\hline 3. Were details of the intervention administered to each group made available? ${ }^{\text {b }}$ & $17(8 \mid .0 \%)$ & $3(14.3 \%)$ & I (4.7\%) \\
\hline 4. Were care providers' experience or skillc in each arm appropriate? ${ }^{\mathrm{d}}$ & $7(33.3 \%)$ & $4(19.1 \%)$ & $10(47.6 \%)$ \\
\hline 5. Was participant (ie, patients) adherence assessed quantitatively? & $6(28.6 \%)$ & $13(61.9 \%)$ & $2(9.5 \%)$ \\
\hline 6. Were participants adequately blinded? & $\mathrm{I}(4.8 \%)$ & $19(90.6 \%)$ & I (4.8\%) \\
\hline \multicolumn{4}{|l|}{ 6.I. If participants were not adequately blinded } \\
\hline $\begin{array}{l}\text { 6.I.I. Were all other treatments and care (ie, cointerventions) the same in } \\
\text { each randomized group? }\end{array}$ & $5(25.0 \%)$ & $12(60.0 \%)$ & $3(15.0 \%)$ \\
\hline 6.I.2. Were withdrawals and lost to follow-up the same in each randomized group? & & 14 (70.0\%) & $6(30.0 \%)$ \\
\hline \multicolumn{4}{|l|}{ 7.I. If care providers were not adequately blinded } \\
\hline $\begin{array}{l}\text { 7.I.I. Were all other treatments and care (ie, cointerventions) the same in each } \\
\text { randomized group? }\end{array}$ & $4(19.1 \%)$ & $10(52.6 \%)$ & $5(26.3 \%)$ \\
\hline 7.I.2. Were withdrawals and lost to follow-up the same in each randomized group? & 0 & II (57.9\%) & $8(42.1 \%)$ \\
\hline 8. Were outcome assessors adequately blinded to assess the primary outcomes? & $2(9.5 \%)$ & $19(90.5 \%)$ & $0(0.0 \%)$ \\
\hline $\begin{array}{l}\text { 8.I. If outcome assessors were not adequately blinded, were specific methods used } \\
\text { to avoid ascertainment bias (systematic differences in outcome assessment)? }\end{array}$ & 0 & $19(100 \%)$ & $0(0 \%)$ \\
\hline 9. Was the follow-up schedule the same in each group?" & $8(38.1 \%)$ & $7(33.3 \%)$ & $6(28.6 \%)$ \\
\hline 10. Were the main outcomes analyzed according to the intention-to-treat principle? & 3 (I4.3\%) & I 8 (85.7\%) & $0(0.0 \%)$ \\
\hline
\end{tabular}

Notes: aFirst and second items were not described in order to RCT design; 'The answer should be "yes" for this item if these data were either described in the report or made available for each arm (reference to a preliminary report, online addendum etc); 'Care provider experience or skill will be assessed only for therapist-dependent interventions (ie, interventions where the success of the treatment are directly linked to care provider's technical skill). For other treatments, this item is not relevant and should be removed from the checklist or answered "unclear"; 'Appropriate experience or skill should be determined according to published data, preliminary studies, guidelines, run-in period, or a group of experts and should be specified in the protocol for each study arm before the beginning of the survey; ${ }^{\mathrm{e} T r e a t m e n t}$ adherence will be assessed only for treatments necessitating interventions (eg, physiotherapy that supposes several sessions, in contrast to a one-shot treatment such as surgery). For one-shot treatments, this item is not relevant and should be removed from the checklist or answered "unclear"; 'The answer should be "yes" for this item, if the main outcome is objective or haed, or if outcomes were assessed by a blinded or at least an independent endpoint review committee, or if outcomes were assessed by an independent outcome assessor trained to perform the measurements in a standardized manner, or if the outcome assessor was blinded to the study purpose and hypothesis; ${ }^{8}$ This item is not relevant for trials in which follow-up is part of the question. For example, this item is not relevant for a trial assessing frequent vs less frequent follow-up for cancer recurrence. In these situations, this item should be removed from the checklist or answered "unclear".

Abbreviation: RCT, randomized controlled trial.

conduct and reporting of the target articles. Our summaries detected omissions of description and nonfulfillments, including detailed information on participants, sample size, assessors, care (health care) providers, blinding, and analysis methods. Furthermore, the description of adverse events and withdrawals were generally insufficient. In the Cochrane Review, ${ }^{1}$ there are strict eligibility criteria for a meta-analysis, and for each article, heterogeneity and low quality of reporting must first be excluded. Because there was insufficient evidence in studies of aquatic exercise, due to poor methodological and reporting quality and heterogeneity, we are unable to offer any conclusions about the effects of aquatic exercise based on an SR.

\section{Characteristics of articles}

The relevant articles represented several studies of locomotorium diseases $(\mathrm{N}=4)$, compared with few studies of respiratory, circulatory, and psychiatric diseases. This characteristic was apparent in the review article ${ }^{10}$ of SRs of RCTs. In that review, there were many articles $(\mathrm{N}=15)$ that targeted the health enhancement effect instead of a specific disease, the primary and/or secondary outcome measurements and target participants varied, and a consistent trend was not detected.

We were interested in comparing not only nonintervention as the control, but also land exercise. There were five reports that defined land exercise as the control group, knee osteoarthritis ${ }^{19,28}$ and rotater cuff tears ${ }^{35}$ as the target disease; the remaining reports ${ }^{22,31}$ were about healthy people. Two studies of knee osteoarthritis did not describe intervention effects. The recent meta-analysis ${ }^{2}$ of RCTs showed no differences between aquatic exercise and land exercise for the painrelieving effects on neurologic or musculoskeletal disease $(P=0.56$; weighted standardized mean difference (SMD), $0.11 ; 95 \%$ confidence interval [CI]: $-0.27-0.50 ; \mathrm{N}=103$ ). Presently, we can not suggest that aquatic exercise is more effective than land exercise for pain relief in locomotorium diseases.

\section{Future research agenda}

There were no studies to clarify the effects on serum lipids, body composition, or blood pressure as the main outcome 
for patients with metabolic syndrome (MS). A joint scientific statement ${ }^{36}$ about MS was issued recently, and many studies based on those criteria or outcomes should be started soon. Studies of aquatic exercise using prevention or cure as the outcome are also expected.

Table 5 shows the future research agenda for aquatic exercise. In advanced nations and areas, it appears that there is interest in studies about mental health as well as MS. Researchers should use the respective checklists for research design and intervention method, which would lead to improvement in the quality of the study, and contribute to the accumulation of evidence. Suitable comparisons are necessary to explain why aquatic exercise is better than other types of dynamic exercise. Aquatic exercise needs a valuable resource (hot water pool), which can not be overlooked in the study feasibility. A recent study ${ }^{37}$ suggested that public health is moving toward the goal of implementing evidence-based interventions, but the feasibility of possible interventions and determining whether comprehensive and multilevel evaluations should be justified to accomplish it.

\section{Study limitations}

This study was based on the PRISMA statement, ${ }^{38}$ except for the meta-analysis. However, there were several limitations to the study. Some selection criteria were common to the studies, as described above; however, bias remained due to differences in eligibility for participation in each study. Publication bias was also a limitation. Since we did not limit our search to English, we found 11 articles (52\%) published in Japanese. Furthermore, we did not check the references of hand-searches and did not contact institutions, societies, specialists known to have expertise in aquatic exercise, and authors of included studies to identify any additional published or unpublished data.

Table 5 Future research agenda on aquatic exercise intervention

\begin{tabular}{|c|c|}
\hline Item & Concrete agenda \\
\hline $\begin{array}{l}\text { Target disease }{ }^{a} \\
\text { or prevention }\end{array}$ & $\begin{array}{l}\text { I. The prevention and curative effect of } \\
\text { metabolic syndrome } \\
\text { 2. The prevention and the curative effect of } \\
\text { mental diseases such as depression }\end{array}$ \\
\hline $\begin{array}{l}\text { Strengthening } \\
\text { of quality }\end{array}$ & $\begin{array}{l}\text { I. Set of research protocol, practice, } \\
\text { description based on each respective checklist } \\
\text { 2. Description of adverse event and withdrawal }\end{array}$ \\
\hline $\begin{array}{l}\text { Feasibility and } \\
\text { intrinsic characteristic }\end{array}$ & $\begin{array}{l}\text { I. Comparison with land exercise and/or } \\
\text { other dynamic intervention } \\
\text { 2. Description of intervention cost }\end{array}$ \\
\hline
\end{tabular}

Note: ${ }^{a}$ The pain-relieving effect of chronic locomotorium diseases has already become clear from many randomized controlled trials.
In terms of quality assessment, disagreements and uncertainties were resolved by discussion between two authors; discussions with a third expert and contact with authors for the purpose of clarification were not allowed.

\section{Conclusion}

Because there was insufficient evidence on aquatic exercise due to poor methodological and reporting quality, and heterogeneity of nRCTs, we were unable to offer any conclusions about the effects of this type of intervention. However, we were able to identify the problems with current nRCTs of aquatic exercise, and propose a strategy of strengthening study quality and stressing the importance of study feasibility as a future research agenda objective.

\section{Acknowledgments}

This study was supported by the Health and Labour Sciences Research Grants (Research on Health Security Control: ID No. H20-007) from the Japanese Ministry of Health, Labour and Welfare in 2009. We would like to express our appreciation to Ms Rie Higashino, Ms Mari Makishi, and Ms Yukiko Yamada for their assistance in this study.

\section{Disclosure}

The authors report no conflicts of interest in this work.

\section{References}

1. Bartels EM, Lund H, Hagen KB, Dagfinrud H, Christensen R, Danneskiold-Samsoe B. Aquatic exercise for the treatment of knee and hip osteoarthritis. Cochrane Database Syst Rev. 2007;(4):CD005523.

2. Hall J, Swinkels A, Briddon J, McCabe CS. Does aquatic exercise relieve pain in adults with neurologic or musculoskeletal disease? A systematic review and meta-analysis of randomized controlled trials. Arch Phys Med Rehabil. 2008;89:873-883.

3. Bender T, Karaglle Z, Balint GP, Gutenbrunner C, Balint PV, Sukenik S. Hydrotherapy, balneotherapy, and spa treatment in pain management. Rheumatol Int. 2005;25:220-224.

4. Yamazaki F, Endo Y, Torii S, Sagawa S, Shiraki K. Continuous monitoring of change in hemodilution during water immersion in humans: effect of water temperature. Aviat Space Environ Med. 2000;71: 632-639.

5. Gabrielsen A, Videbaek R, Johansen LB, et al. Forearm vascular and neuroendocrine responses to graded water immersion in humans. Acta Physio Scand. 2000;169:87-94.

6. Fam AG. Spa treatment in arthritis: a rheumarologist's view. J Rheumatol. 1991;18:1775-1777.

7. Kamioka H, Nakamura Y, Okada S, et al. Effectiveness of comprehensive health education combining lifestyle education and hot spa bathing for male white-collar employees: a randomized controlled trial with 1-year follow-up. J Epidemiol. 2009;19:219-230.

8. Kamioka H, Nakamura Y, Yazaki T, et al. Effectiveness of comprehensive health education combining hot spa bathing and lifestyle education in middle-aged and elderly women: one-year follow-up on randomized controlled trial of three- and six-month interventions. J Epidemiol. 2006; $16: 35-44$. 
9. Kamioka H, Ohshiro H, Mutoh Y, et al. Effect of long-term comprehensive health education on the elderly in a Japanese village: Unnan cohort study. Int J Sports Health Sci. 2008;6:60-65.

10. Kamioka H, Tsutani K, Okuizumi H, et al. Effectiveness of aquatic exercise and balneotherapy: a summary of systematic reviews based on randomized controlled trials of water immersion therapies. J Epidemiol. 2010;20:2-12.

11. Pittler MH, Karagülle MZ, Karagülle M, Ernst E. Spa therapy and balneotherapy for treating low back pain: meta-analysis of randomized trials. Rheumatol. 2006;45:880-884.

12. Jarlais DC, Lyles $C$, Crepaz $N$; for the TREND group. Improving the reporting quality of nonrandomized evaluations of behavioral and public health interventions. Am J Public Health. 2004;94:361-366.

13. Boutron I, Moher D, Tugwell P, et al. A checklist to evaluate a report of a nonpharmacological trial (CLEAR NPT) was developed using consensus. J Clin Epidemiol. 2005;58:1233-1240.

14. Atkins D, Best D, Briss PA, et al. Grading quality of evidence and strength of recommendations. BMJ. 2004;328:1490-1497.

15. Willén C, Sunnerhagen KS, Grimby G. Dynamic water exercise in individuals with late poliomyelitis. Arch Phys Med Rehabil. 2001;82: 66-72.

16. Ebisu T, Kobayashi M, Katsuki T, Yanagimoto Y. Effectiveness of serum lipids on spa-walking [in Japanese]. Health Care. 2001;43: 981-984

17. Aoba T, Matsumoto T, Sugano A, Nomura T. The effects of enforcement water exercise class on hypotensive to blood pressure at elderly subjects [in Japanese with English abstract]. Ann Reports Health Phys Educ Sports Sci. 2001;20:99-104.

18. Yamada T, Watanabe E, Okada A, Takeshima N. Effects of water-based well-rounded exercise on vital age and physical fitness in older adults [in Japanese with English abstract]. Jpn Soc Phys Anthropol. 2002;7: 87-93.

19. Murai E, Takeda Y, Imaya T, Uchiyama E, Nakazono K, Nakajima H. Exercise therapy for osteoarthritis of the knee -preliminarily study of water exercise [in Japanese with English abstract]. JJpn Soc Clin Sports Med. 2002;10:54-60.

20. Igarashi Y, Kondo T, Yamaoka K, et al. Health effect of aquatic exercise therapy using a hot spring [in Japanese]. Sick Body Phys. 2002;36: 11-14.

21. Pechter Ü, Ots M, Mesikepp S, et al. Beneficial effects of water-based exercise in patients with chronic kidney disease. Int $J$ Rehabil Res. 2003;26:153-156.

22. Douris P, Southard V, Varga C, Schauss W, Gennaro C, Reiss A. The effect of land and aquatic exercise on balance scores in older adules. J Geriatr Phys Ther. 2003;26:3-6.

23. Liquori A, Widener G, Clark L. Effects of a 6-week prenatal water exercise program on physiological parameters and well-being in women with pregnancies in the 2 nd-3rd trimesters: a pilot study. J Section Women's Health. 2003;27:11-19.

24. Lin SY-C, Davey RC, Cochrane T. Community rehabilitation for older adults with osteoarthritis of the lower limb: a controlled clinical trial. Clin Rehabil. 2004;18:92-101.
25. Akamine T, Yamanaka T, Taguchi N, et al. Effects of underwater exercise with hot spring bathing on middle aged people [in Japanese with English abstract]. Kyushu Yamaguchi Sports Sci J. 2005;17:7-12.

26. Takumi Y, Moriya K, Oda S, Adikari MO, Fukuoka E. Participation in an aquatic exercise class twice a week for 12 weeks improved physical fitness for good walking, walking self-efficacy on winter roads, mental health and QOL in elderly women [in Japanese with English abstract] Jpn J Biometeor. 2005;42:17-27.

27. Takumi Y, Moriya K. Participation in an aquatic walking class for 12 weeks improved physical fitness for good walking, emotion and walking self-efficacy on winter roads in middle-aged and elderly women [in Japanese with English abstract]. Jpn J Biometeor. 2005;42:5-15.

28. Lee HY. Comparison of effects among Tai-chi exercise, aquatic exercise, and a self-help program for patients with knee osteoarthritis [in Korean with English abstract]. J Korean Acad Nurs. 2006;36:571-580.

29. Lord SR, Matters B, George RS, Thomas M, Bindon J, Chan DK-Y. The effects of water exercise on physical functioning in older people. Australasian J Ageing. 2006;25:36-41.

30. Smith SA, Michel Y. A pilot study on the effects of aquatic exercises on discomforts of pregnancy. J Obstet Gynecol Neonatal Nurs. 2006;35:315-323

31. Chishaki A, Makaya M, Nagahiro C, et al. Comparison of usefulness between water exercise and gymnastic exercise, Jikyojyutsu, in maintaining physical ability for the elderly [in Japanese with English abstract]. Memoirs Dep Health Sci Sch Med Kyushu Univ. 2006;7: 23-32

32. Kawasaki T, Muratani H, Ozoe N, Higaki H, Kawasaki J. The effect of aquatic exercise on preventing falls and life-style related disease among middle-aged and elderly people [in Japanese]. Jpn J Clin Exper Med. 2007;84:402-411.

33. Nishikawa A, Ueda Y, Wakayama K, Hayashi Y. Effect of aquatic walking on the cardiovascular patients in our hospital and health-related QOL- in comparison with indoor exercise therapy [in Japanese]. JJCR. 2008; 13:135-138.

34. Rotstein A, Harush M, Vaisman N. The effect of a water exercise program on bone density of postmenopausal women. J Sports Med Phys Fitness. 2008;48:352-359.

35. Brady B, Redfern J, Macdougal G, Williams J. The addition of aquatic therapy to rehabilitation following surgical rotator cuff repair: a feasibility study. Physiother Res Int. 2008;13:153-161.

36. Alberti KGMM, Eckel RH, Grundy SM, et al. Harmonizing the metabolic syndrome: a joint interim statement of the International Diabetes Federation TaskForce on Epidemiology and Prevention; National Heart, Lung, and Blood Institute; American Heart Association; World Heart Federation; International Atherosclerosis Society; and International Association for the Study of Obesity. Circulation. 2009;120:1640-1645.

37. Bowen DJ, Kreuter M, Spring B, et al. How we design feasibility studies. Am J Prev Med. 2009;36:452-457.

38. Liberati A, Altman DG, Tetzlaff J, et al. The PRISMA statement for reporting systematic reviews and meta-analyses of studies that evaluate health care interventions: explanation and elaboration. Ann Intern Med. 2009;151:W65-W94. 
Appendix I References to studies excluded from this review

\begin{tabular}{|c|c|c|c|}
\hline No. & Author. Journal (Year) & Title & Reason for exclusion \\
\hline EI & Bar-Or. Phys Sport Med (2000) & Juvenile obesity, physical activity, and lifestyle changes & Review article \\
\hline E2 & $\begin{array}{l}\text { Biering-Sørensen et al. } \\
\text { Spinal Cord (2000) }\end{array}$ & $\begin{array}{l}\text { Bacterial contamination of bath-water from spinal cord } \\
\text { lesioned patients with pressure ulcers exercising in the water }\end{array}$ & $\begin{array}{l}\text { No intervention by aquatic } \\
\text { exercise }\end{array}$ \\
\hline E3 & $\begin{array}{l}\text { Darby et al. J Sports Med } \\
\text { Phys Fitness (2000) }\end{array}$ & $\begin{array}{l}\text { Physiological responses during two types of exercise } \\
\text { performed on land and in the water }\end{array}$ & Cross-over design \\
\hline E4 & Hsieh. MAMM (2000) & Aquatic exercise makes a splash & Program idea \\
\hline E5 & Jamison. Rehab Management (2000) & Long-term rehab. The healing properties of water & Review article \\
\hline E6 & $\begin{array}{l}\text { Sugano et al. J Physiol Anthropol } \\
\text { Appl Hum Sci (2000) }\end{array}$ & $\begin{array}{l}\text { Influence of water exercise and land stretching } \\
\text { on salivary cortisol concentrations and anxiety in } \\
\text { chronic low back pain patients }\end{array}$ & Cross-over design \\
\hline E7 & $\begin{array}{l}\text { Watanabe et al. Percept } \\
\text { Mot Skills (2000) }\end{array}$ & $\begin{array}{l}\text { Comparison of water- and land-based exercise in the } \\
\text { reduction of state anxiety among older adults }\end{array}$ & $\mathrm{RCT}$ \\
\hline E8 & Alzugaray. Midwifery Today (200I) & $\begin{array}{l}\text { American AquaNatal: a midwifery-based holistic prenatal } \\
\text { water exercise and educational program }\end{array}$ & Program idea \\
\hline E9 & $\begin{array}{l}\text { Hartmann et al. Geburtsh } \\
\text { Frauenheilk (200I) }\end{array}$ & $\begin{array}{l}\text { "Aqua-Fit" during pregnancy: Maternal and fetal hemodynamic } \\
\text { responses during rest, immersion and exercise }\end{array}$ & No control group \\
\hline EIO & $\begin{array}{l}\text { Piso et al. Phys Med Rehabil } \\
\text { Kuror (200I) }\end{array}$ & Analgesic effects of sauna in fibromyalgia & $\mathrm{RCT}$ \\
\hline EII & Postollec. Back on Track (200I) & $\begin{array}{l}\text { Back on Track Aquatic therapy can help patients } \\
\text { with back pain resume normal activity }\end{array}$ & Program idea \\
\hline EI2 & $\begin{array}{l}\text { Shono et al. J Physiol } \\
\text { Anthropol (200I) }\end{array}$ & $\begin{array}{l}\text { Physiological responses to water-walking } \\
\text { in middle aged women }\end{array}$ & $\begin{array}{l}\text { Physiological responses } \\
\text { (regulatory experiment group } \\
\text { versus the other group) }\end{array}$ \\
\hline EI3 & $\begin{array}{l}\text { Watanabe et al. Percept } \\
\text { Mot Skills (200I) }\end{array}$ & $\begin{array}{l}\text { Effects of increasing expenditure of energy during exercise } \\
\text { on psychological well-being in older adults }\end{array}$ & No control group \\
\hline $\mathrm{EI} 4$ & $\begin{array}{l}\text { Seki et al. Bul of Nurs College, } \\
\text { Wakayama Med Univ (200I) }\end{array}$ & $\begin{array}{l}\text { The effects of maintaining health by exercising in the } \\
\text { water in middle to old-aged women }\end{array}$ & No control group \\
\hline EI5 & Belza et al. Nus Res (2002) & Does adherence make a difference & $\mathrm{RCT}$ \\
\hline EI6 & $\begin{array}{l}\text { Huey et al. Best of Both } \\
\text { Worlds (2002) }\end{array}$ & $\begin{array}{l}\text { Combining aquatics with land exercise effectively } \\
\text { reduces back pain }\end{array}$ & Program idea \\
\hline EI7 & $\begin{array}{l}\text { V. Kendrick et al. J Aquatic } \\
\text { Phys Ther (2002) }\end{array}$ & $\begin{array}{l}\text { Effects of water exercise on improving muscular strength } \\
\text { and endurance in suburban and inner city older adults }\end{array}$ & Cohort study \\
\hline EI8 & Warden et al. Sports Med (2002) & Aetiology of rib stress fractures in rowers & Review article \\
\hline EI9 & Weinsier et al. An J Clin Nutr (2002) & $\begin{array}{l}\text { Free-living activity energy expenditure in women successful } \\
\text { and unsuccessful at maintaining a normal body weight }\end{array}$ & $\begin{array}{l}\text { No intervention by } \\
\text { aquatic exercise }\end{array}$ \\
\hline E20 & $\begin{array}{l}\text { Winter et al. J Aquatic } \\
\text { Phys Ther (2002) }\end{array}$ & $\begin{array}{l}\text { Effects of aquatic lumbar stabilization and strengthening } \\
\text { exercise protocol on chronic low back pain patients }\end{array}$ & No control group \\
\hline E2I & $\begin{array}{l}\text { Sudo et al. Ann reports Health } \\
\text { Phys Educ Sports Sci (2002) }\end{array}$ & $\begin{array}{l}\text { Effects of the underwater exercise on elderly } \\
\text { with low-muscle strength }\end{array}$ & Cross-sectional study \\
\hline E22 & $\begin{array}{l}\text { Tsushita et al. J Aquatic Health } \\
\text { Med (2002) }\end{array}$ & $\begin{array}{l}\text { Effect of aquatic exercise on lifestyle-related diseases } \\
\text { in middle-aged women }\end{array}$ & No control group \\
\hline E23 & $\begin{array}{l}\text { Campbell et al. Med Sci } \\
\text { Sports Exer (2003) }\end{array}$ & $\begin{array}{l}\text { Metabolic and cardiovascular response to shallow } \\
\text { water exercise in young and older women }\end{array}$ & Physiological responses \\
\hline E24 & $\begin{array}{l}\text { Charitopoulos et al. J Human } \\
\text { Mov Stud (2003) }\end{array}$ & $\begin{array}{l}\text { Application of an aqua exercise programme in children } \\
\text { with exercise induced asthma }\end{array}$ & No control group \\
\hline E25 & $\begin{array}{l}\text { Driver et al. J Cognit } \\
\text { Rehabil (2003) }\end{array}$ & $\begin{array}{l}\text { Effect of an aquatics program on psycho/social experiences } \\
\text { of individuals with brain injuries: a pilot study }\end{array}$ & Case report \\
\hline E26 & $\begin{array}{l}\text { Gyurcsik et al. } \\
\text { Arthritis Rheum (2003) }\end{array}$ & $\begin{array}{l}\text { Exercise-related goals and self-efficacy as correlates } \\
\text { of aquatic exercise in individuals with arthritis }\end{array}$ & Cross-sectional study \\
\hline E27 & Mayo Clin. Health Lett (2003) & Water exercise for arthritis: low-impact fitness & Health letter \\
\hline E28 & $\begin{array}{l}\text { Suomi et al. Arch Phys } \\
\text { Med Rehabil (2003) }\end{array}$ & $\begin{array}{l}\text { Effects of arthritis exercise programs on functional } \\
\text { fitness and perceived activities of daily living measures } \\
\text { in older adults with arthritis }\end{array}$ & RCT \\
\hline E29 & $\begin{array}{l}\text { Weidner et al. Aktuelle } \\
\text { Rheumatoligie (2003) }\end{array}$ & Rheumatism and Sports & Review article \\
\hline $\mathrm{E} 30$ & $\begin{array}{l}\text { Yurtkuran et al. Am J Phys } \\
\text { Med Rehabil (2003) }\end{array}$ & $\begin{array}{l}\text { Evaluation of hormonal response and ultrasonic changes in heel } \\
\text { bone by aquatic exercise in sedentary postmenopausal women }\end{array}$ & $\mathrm{RCT}$ \\
\hline
\end{tabular}

(Continued) 
Appendix I (Continued)

\begin{tabular}{|c|c|c|c|}
\hline No. & Author. Journal (Year) & Title & Reason for exclusion \\
\hline E3I & $\begin{array}{l}\text { Sugano et al. Bull Inst Health } \\
\text { Sport Sci, Univ of Tsukuba (2003) }\end{array}$ & $\begin{array}{l}\text { Prescription of water exercise for low back pain patients. Influence } \\
\text { of water exercise and land stretching on salivary cortisol } \\
\text { concentrations and anxiety in chronic low back pain patients }\end{array}$ & Study report \\
\hline E32 & $\begin{array}{l}\text { Matsumoto et al. Ann Reports } \\
\text { Health Physl Educ Sports Sci (2003) }\end{array}$ & $\begin{array}{l}\text { Blood chemical values in elderly persons performing } \\
\text { habitual aquatic exercise }\end{array}$ & Cross-sectional study \\
\hline E33 & $\begin{array}{l}\text { Benelli et al. J Strength } \\
\text { Cond Res (2004) }\end{array}$ & $\begin{array}{l}\text { Physiological responses to fitness activities: a comparison } \\
\text { between land-based and water aerobics exercise }\end{array}$ & Cross-over design \\
\hline E34 & Booth. Activ Adapt Aging (2004) & $\begin{array}{l}\text { Water exercise and its effect on balance and gait to reduce } \\
\text { the risk of falling in older adults }\end{array}$ & Cross-sectional study \\
\hline E35 & $\begin{array}{l}\text { Preis et al. Fisioterapia } \\
\text { em Movimento (2004) }\end{array}$ & $\begin{array}{l}\text { Análise de um programa de execução de saltos múltiplos } \\
\text { verticais no meio líquido e no solo }\end{array}$ & Biomechanical trial \\
\hline E36 & $\begin{array}{l}\text { Roebrs et al. J Neurol } \\
\text { Phys Ther (2004) }\end{array}$ & $\begin{array}{l}\text { Effects of an aquatics exercise program on quality of life } \\
\text { measures for individuals with progressive multiple sclerosis }\end{array}$ & No control group \\
\hline E37 & $\begin{array}{l}\text { Aoba et al. Kokushikan Soc } \\
\text { Sport Sci (2004) }\end{array}$ & The effect of continuation term on BP at water exercise & $\begin{array}{l}\text { No retrospective comparison } \\
\text { between aquatic exercise } \\
\text { group and drop out group of } \\
\text { aquatic exercise }\end{array}$ \\
\hline E38 & $\begin{array}{l}\text { Ashida et al. Ann Reports Misasa } \\
\text { Med Center, Okayama Univ (2004) }\end{array}$ & $\begin{array}{l}\text { Clinical study on reduction of costs of drugs for the } \\
\text { treatment of asthma in relation to the administration method }\end{array}$ & Not intervention study \\
\hline E39 & $\begin{array}{l}\text { Katayama et al. Descente } \\
\text { Sports Sci (2004) }\end{array}$ & $\begin{array}{l}\text { Effects of weight reduction through dietary restriction and } \\
\text { exercise training on blood fluidity in obese middle-aged women }\end{array}$ & $\begin{array}{l}\text { No intervention by aquatic } \\
\text { exercise }\end{array}$ \\
\hline E40 & $\begin{array}{l}\text { Higuma et al. } \\
\text { J Oita Med Assoc (2004) }\end{array}$ & Rehabilitation after TKA in a hospital & Cross-sectional study \\
\hline E4I & $\begin{array}{l}\text { Barry Dale et al. } \\
\text { Rehabil Management (2005) }\end{array}$ & $\begin{array}{l}\text { Aquatic therapy offers benefits to a wide } \\
\text { range of clinical populations }\end{array}$ & Review article \\
\hline E42 & $\begin{array}{l}\text { Cider et al. Clin Physiol } \\
\text { Funct Imaging (2005) }\end{array}$ & $\begin{array}{l}\text { Cardiorespiratory effects of warm water immersion } \\
\text { in elderly patients with chronic heart failure }\end{array}$ & $\begin{array}{l}\text { Comparison between patients } \\
\text { on healthy people }\end{array}$ \\
\hline E43 & $\begin{array}{l}\text { Kelly et al. Development } \\
\text { Med Child Neurol (2005) }\end{array}$ & Aquatic exercise for children with cerebral palsy & Review article \\
\hline E44 & $\begin{array}{l}\text { Kato et al. Research Reports } \\
\text { Suzuka Univ Med Sci Technol (2005) }\end{array}$ & $\begin{array}{l}\text { Influence of aquatic-exercise on arterial blood pressure } \\
\text { and acceleration plethysmogram in middle-aged women }\end{array}$ & Case report \\
\hline E45 & $\begin{array}{l}\text { Matsubara et al. } \\
\text { Ishikawa J Nurs (2005) }\end{array}$ & $\begin{array}{l}\text { Case-control study on long-term effect of programmed } \\
\text { movement practice course combined with bathing in hot spring }\end{array}$ & Case-control study \\
\hline E46 & Gusi et al. Arthritis Rheum (2006) & $\begin{array}{l}\text { Exercise in waist-high warm water decreases pain and improves } \\
\text { health-related quality of life and strength in lower extremities } \\
\text { in women with fibromyalgia }\end{array}$ & $\mathrm{RCT}$ \\
\hline E47 & Katia et al. JAGS (2006) & $\begin{array}{l}\text { Older people involved in physical activity benefit from } \\
\text { water exercise, showing longer total sleep time }\end{array}$ & Letter to the journal's editor \\
\hline E48 & $\begin{array}{l}\text { Goto et al. J Jpn Assoc Phys } \\
\text { Med Balneol Climatol (2006) }\end{array}$ & $\begin{array}{l}\text { The effect of Spa-aqua therapy on lifestyle-related diseases } \\
\text { in collaboration with public spa facility }\end{array}$ & No control group \\
\hline E49 & Kokubu et al. Hip Joint (2006) & Effect of underwater exercise for hip joint disease & Cross-sectional study \\
\hline E50 & $\begin{array}{l}\text { Yano et al.Jpn J Phys } \\
\text { Fitness Sports Med (2006) }\end{array}$ & $\begin{array}{l}\text { Effect of fluid in ingestion on physiological response before } \\
\text { walking in a pool }\end{array}$ & $\begin{array}{l}\text { No intervention by aquatic } \\
\text { exercise }\end{array}$ \\
\hline E5I & Brody. J Aquatic Phys Ther (2007) & Aquatic physical therapy practice analysis & Practice analysis \\
\hline E52 & Goodwin. OT Practice (2007) & $\begin{array}{l}\text { Exploring the effects of a swim program for clients } \\
\text { with down syndrome }\end{array}$ & No control group \\
\hline E53 & Kang et al. J Gerontol Nurs (2007) & Aquatic exercise in older Korean women with arthritis & No control group \\
\hline E54 & Kron. Complementary Med (2007) & Water therapies & Review article \\
\hline E55 & McManus B. Pedia Phys Ther (2007) & $\begin{array}{l}\text { The effect of aquatic therapy on functional mobility } \\
\text { of infants and toddlers in early intervention }\end{array}$ & $\mathrm{RCT}$ \\
\hline E56 & $\begin{array}{l}\text { Magkos et al. } \\
\text { Clin J Sports Med (2007) }\end{array}$ & $\begin{array}{l}\text { The bone response to nonweight-bearing exercise } \\
\text { is sport-, site-, and sex-specific }\end{array}$ & Cross-sectional study \\
\hline E57 & Schmid et al. Heart (2007) & $\begin{array}{l}\text { Influence of water immersion, water gymnastics } \\
\text { and swimming on cardiac output in patients with heart failure }\end{array}$ & $\begin{array}{l}\text { Physiological responses of } \\
\text { various performances in } \\
\text { water }\end{array}$ \\
\hline E58 & Alpert. Cardiology (2008) & $\begin{array}{l}\text { A water-based exercise program for patients with } \\
\text { coronary artery disease }\end{array}$ & Editorial comment \\
\hline
\end{tabular}




\section{Appendix I (Continued)}

\begin{tabular}{|c|c|c|c|}
\hline No. & Author. Journal (Year) & Title & Reason for exclusion \\
\hline E59 & $\begin{array}{l}\text { Hujibregts et al. } \\
\text { Top Stroke Rehabil (2008) }\end{array}$ & $\begin{array}{l}\text { Implementation, process, and preliminary outcome } \\
\text { evaluation of two community programs for persons } \\
\text { with stroke and their care partners }\end{array}$ & $\begin{array}{l}\text { Not intervention by aquatic } \\
\text { exercise }\end{array}$ \\
\hline E60 & $\begin{array}{l}\text { Kelley et al. Therapeutic } \\
\text { Recreation J (2008) }\end{array}$ & $\begin{array}{l}\text { Comparing the effects of aquatic and land-based exercise } \\
\text { on the physiological stress response of women with fibromyalgia }\end{array}$ & $\begin{array}{l}\text { A single-subject alternating } \\
\text { treatment design }\end{array}$ \\
\hline E6I & $\begin{array}{l}\text { Tokmakidis et al. } \\
\text { Cardiology (2008) }\end{array}$ & $\begin{array}{l}\text { Training, detraining and retraining effects after a water-based } \\
\text { exercise program in patients with coronary artery disease }\end{array}$ & $\mathrm{RCT}$ \\
\hline E62 & $\begin{array}{l}\text { Colado et al. } \\
\text { Eur J Appl Physiol (2009) }\end{array}$ & $\begin{array}{l}\text { Effects of aquatic resistance training on health and fitness } \\
\text { in postmenopausal women }\end{array}$ & $\mathrm{RCT}$ \\
\hline E63 & $\begin{array}{l}\text { Fragala-Pinkham et al. } \\
\text { Pedia Phys Ther (2009) }\end{array}$ & $\begin{array}{l}\text { An aquatic physical therapy program at a pediatric } \\
\text { rehabilitation hospital: a case series }\end{array}$ & Case report \\
\hline E64 & $\begin{array}{l}\text { Laurent et al. } \\
\text { Euro Soc Cardiol (2009) }\end{array}$ & $\begin{array}{l}\text { Training-induced increase in nitric oxide metabolites } \\
\text { in chronic heart failure and coronary artery disease: } \\
\text { an extra benefit of water-based exercises? }\end{array}$ & $\mathrm{RCT}$ \\
\hline E65 & $\begin{array}{l}\text { Schmid et al. } \\
\text { Heart (2009) }\end{array}$ & $\begin{array}{l}\text { Influence of water immersion, water gymnastics and swimming } \\
\text { on cardiac output in patients with heart failure }\end{array}$ & $\begin{array}{l}\text { No intervention by aquatic } \\
\text { exercise }\end{array}$ \\
\hline E66 & $\begin{array}{l}\text { Souza Vale et al. } \\
\text { Arch Gerontol Geriatrics (2009) }\end{array}$ & $\begin{array}{l}\text { Effects of muscle strength and aerobic training on basal } \\
\text { serum levels of IGF-I and cortisol in elderly women }\end{array}$ & $\begin{array}{l}\text { No intervention by aquatic } \\
\text { exercise }\end{array}$ \\
\hline E67 & $\begin{array}{l}\text { Tolomio et al. } \\
\text { Clin Ter (2009) }\end{array}$ & $\begin{array}{l}\text { Effects of a combined weight- and non weight-bearing (water) } \\
\text { exercise program on bone mass and quality in postmenopausal } \\
\text { women with low bone mineral density }\end{array}$ & $\begin{array}{l}\text { No intervention by aquatic } \\
\text { exercise }\end{array}$ \\
\hline
\end{tabular}

Abbreviations: IGF-I, insulin-like growth factor-I; RCT, randomized controlled trial.

\section{Publish your work in this journal}

The International Journal of General Medicine is an international, peer-reviewed open-access journal that focuses on general and internal medicine, pathogenesis, epidemiology, diagnosis, monitoring and treatment protocols. The journal is characterized by the rapid reporting of reviews, original research and clinical studies across all disease areas.
A key focus is the elucidation of disease processes and management protocols resulting in improved outcomes for the patient.The manuscript management system is completely online and includes a very quick and fair peer-review system. Visit http://www.dovepress.com/ testimonials.php to read real quotes from published authors. 